\title{
natin \\ CFD Simulation and Optimization of Adiabatic Reaction Process of C-2 Hydrogenation
}

\author{
Wenjing Wei \\ Key Laboratory of Advanced Control and Optimization for Chemical Processes, Ministry of Education, East \\ China University of Science and Technology, Shanghai 200237, China; 13162215880@163.com
}

\begin{abstract}
In this paper, the computational fluid dynamics (CFD) method has been used to simulate the gas flow and the catalytic hydrogenation process of the C-2 hydrogenation adiabatic reactor in a practical ethylene production unit of a chemical plant. The laminar finite-rate model was used for the chemical reaction model. The standard $k-\varepsilon$ two-equation model was used for the turbulence model. The exponent-function kinetic model was used for the reaction dynamics model. The simulation results were in good agreement with the actual industrial data obtained from the C-2 hydrogenation reactor, and the distributions of reaction parameters such as temperature and component concentrations in the reactor were further analyzed. Based on the validated CFD models, the structure and operating conditions of the reactor, as well as hydrogen load distribution ratios, are simulated for comparing the comprehensive performance metrics of ethylene selectivity and acetylene conversion rate; as a result, we can obtain the optimal temperature of $312 \mathrm{~K}$, the optimal hydrogen/acetylene ratio of 0.10 as well as the optimal hydrogen load distribution ratio of 4:4:2, and the diameter/height ratio exerts a marginal effect on the selectivity, conversion rate and harmonic mean of the reactor.
\end{abstract}

Keywords: C-2 hydrogenation adiabatic reactor; mathematical model; CFD simulation; optimization

\section{Introduction}

In this paper, the simulation and optimization of the adiabatic reactor of C-2 hydrogenation in a 1 million tons ethylene production unit of a chemical plant are taken as the research background. The process flow of C-2 hydrogenation reaction is shown in Figure 1. The top gas of deethanizer is preheated by C-2 hydrogenation reactor discharge and low-pressure steam, and then mixed with hydrogen, and finally passed through the first catalyst bed. The bed temperature rise is proportional to the amount of hydrogen added into the feed. The reactor system has a safety interlock system which can shut off hydrogen when the reactor temperature rises excessively. The discharge from the first bed is mixed with hydrogen again and cooled with cooling water. After passing through the green oil separation tank, it enters the second bed. The discharge from the second bed is mixed with hydrogen again and cooled with cooling water. After passing through the second green oil separation tank, it enters the third bed to complete acetylene conversion. The acetylene concentration in the discharge from the third bed is lower than $1 \mathrm{~mL} / \mathrm{m}^{3}$, and the discharge is cooled by cooling water and performed the heat exchange with the feed of $\mathrm{C}-2$ hydrogenation reactor. If necessary, hydrogen rich in $\mathrm{CO}$ (CO is the moderator for acetylene hydrogenation) is injected into the reactor inlet. 


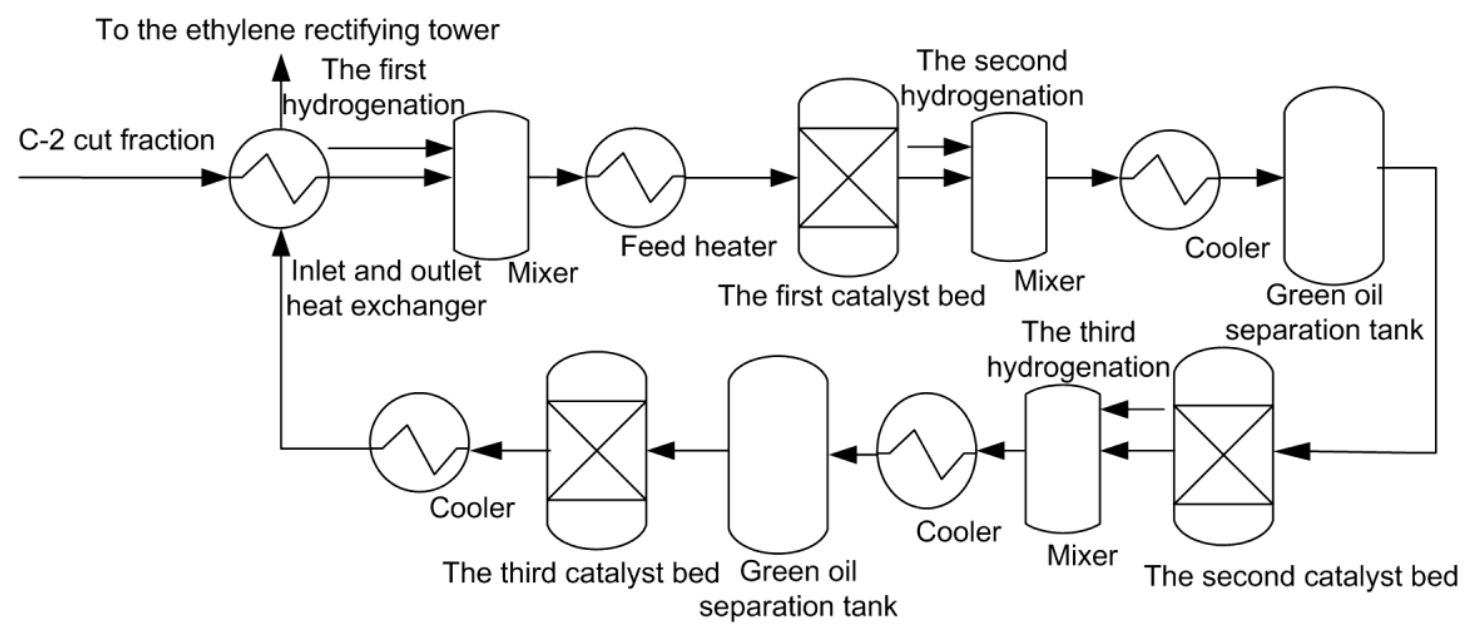

Figure 1. A process flow of C-2 hydrogenation reaction.

In many chemical products, the concentration of acetylene must be reduced to a specified very small concentration. For example, in polymer-grade ethylene products, the concentration of acetylene must be lower than $2 \mathrm{~mL} / \mathrm{m}^{3}$. Otherwise, the presence of acetylene will cause serious harm to the entire reaction device and the product. For instance, the presence of acetylene in the high-density polyethylene (HDPE) generator will seriously affect the activity of the catalyst, thus reducing the performance of the entire polymerization reaction. In the production of low-density polyethylene, the presence of acetylene in large quantity will seriously reduce the antioxidant properties of the products. Because of the great harm of acetylene, in C-2 hydrogenation reaction, we must control the concentration of acetylene within a reasonable range to ensure that the subsequent process can proceed smoothly.

C-2 hydrogenation plays a significant role in the whole ethylene production process. The presence of acetylene in ethylene will directly affect the activity of the catalyst of subsequent polymerization reaction, and thus affect the quality and yield of polymerized ethylene products. C-2 hydrogenation is the most widely used method to remove acetylene in the industry. It converts acetylene to ethylene or ethane to remove acetylene by catalytic hydrogenation reaction.

Because C-2 hydrogenation plays a significant role in the whole ethylene production process, many researchers at home and abroad have conducted in-depth research and exploration on the simulation of C-2 hydrogenation and obtained a series of research achievements. For example, Tian et al. (2016) [1] first used computational fluid dynamics (CFD) to comprehensively simulate the industrial adiabatic and isothermal reactor, and obtained the distribution of gas velocity, temperature and concentration in the reactor. The simulation results were in close agreement with the actual industrial data, which verified the accuracy of the model. The geometric structure and operating parameters of the reactor were optimized by using the obtained model, and the maximum ethylene selectivity was obtained. Azizi et al. (2013) [2] simulated industrial acetylene hydrogenation units using three available kinetic models. The simulation results were compared with the experimental data of six days, and the best model was selected. In addition, the effect of feed temperature and the amount of hydrogen injected on the ethylene selectivity was also studied, and a new method was proposed to improve the hydrogenation process and reduce unwanted by-products. In the simulation of the improved process, the temperature, hydrogen flow rate and $\mathrm{H}-1 / \mathrm{H}-2$ ratio of the hydrogenation reactor were taken as the adjustable parameters of the process optimization. An improved least variance constrained control method was proposed by $\mathrm{Xu}$ and Zhao (2015) [3]. The final effective feedback rate was obtained by using LMI (linear matrix inequality). Under the condition that the acetylene concentration at the outlet was lower than $5 \mathrm{ppm}$, the purpose of improving the activity and selectivity of the catalyst was realized, and the excessive hydrogenation was avoided. In the literature Lu et al. (2016) [4], in view of the short operating cycle and decreased hydrogenation selectivity caused by the reduction of the cracking degree of the ethylene plant, the original three-stage hydrogenation process was changed to two-stage hydrogenation, and the 
reaction conditions were simulated and optimized. The purpose of improving the conversion rate of acetylene and the total selectivity of ethylene was achieved, and the long-term stable operation of the catalyst was ensured. Zhang and Wang (2003) [5] utilized Aspen software to simulate the C-2 hydrogenation reactor, and obtained the optimal reaction temperature of acetylene selective hydrogenation, thus improving the ethylene production and catalyst regeneration cycle, and also conducive to the stable operation of the unit. However, the effect of hydrogenation amount on the ethylene selectivity has not been investigated in the literature. Therefore, the operational parameters of hydrogenation reactor have not been comprehensively analyzed and optimized. Yang et al. (2011) [6] optimized and adjusted the main parameters of the C-2 hydrogenation reactor, improving the selectivity of the catalyst, extending the regeneration cycle of the reactor, and achieving the purpose of reducing production cost and energy consumption. Zhong (2005) [7] proposed a method to quickly evaluate the selectivity of the catalyst. The selectivity of the catalyst could be obtained by only knowing the conversion amount of acetylene and the bed temperature difference. By applying this method to three different catalysts of C-2 hydrogenation in an ethylene cracking unit, the selectivity of the catalyst could be calculated quickly, and the operation of C-2 hydrogenation process could be guided online and in real time.

In addition, many scholars have studied the effects of operating parameters such as temperature, hydrogen/acetylene ratio and $\mathrm{CO}$ concentration on the performance of the catalyst. For example, Peng (2014) [8] analyzed the influence of operating parameters of the C-2 hydrogenation reactor, such as temperature, hydrogen/acetylene ratio, CO concentration, load distribution, etc., on the hydrogenation selectivity of the catalyst, while other reaction parameters, such as reaction pressure, space velocity, acetylene concentration, etc., also had certain influences on the hydrogenation reaction, but could not be utilized as a regulation means. Che et al. (2013) [9] used the front-end hydrogenation process in the C-2 hydrogenation reactor, and the influences of process parameters such as inlet temperature, space velocity and $\mathrm{CO}$ concentration on the catalyst performance were compared. The results showed that the inlet temperature had the greatest influence on the catalyst performance, followed by the influence of $\mathrm{CO}$ and the space velocity had the least influence. Dong (2014) [10] studied the influence of operating parameters such as inlet temperature, space velocity, $\mathrm{CO}$ and $\mathrm{C}_{4}$ concentration on the performance of the catalyst. By optimizing these operating parameters, the C-2 hydrogenation reactor was maintained at the stable operation. Huang (2016) [11] ensured the safety, stability and long-term operation of the C-2 hydrogenation reactor by optimizing the inlet $\mathrm{CO}$ concentration and inlet temperature and controlling the impurity content in the feedstock. The acetylene converter in the literature $\mathrm{Gu}$ et al. (2017) [12] adopted the front-end hydrogenation process, and the concentration of $\mathrm{CO}$ in the pyrolysis gas had a certain influence on the selectivity and activity of the catalyst, resulting in the decrease of ethylene selectivity, and the concentration of outlet acetylene could not meet the requirements. Some measures, such as vulcanization treatment, could reduce the use of alcohol and ether in the cracking feedstock, which could effectively control the generation of $\mathrm{CO}$. By adjusting methanol pressure and reactor inlet temperature, the influence of $\mathrm{CO}$ concentration fluctuation on the reactor could be eliminated. Jiang et al. (2002) [13] optimized and controlled the operating parameters of the C-2 hydrogenation reactor, such as $\mathrm{CO}$ injection amount, reaction temperature, hydrogen/acetylene ratio, load distribution, etc., which effectively reduced the production of green oil, extended the operating cycle of the reactor, and significantly improved the hydrogenation selectivity and ethylene increment.

In addition, many researchers employ chemical process simulation software, such as Fluent, Aspen Plus, HYSYS etc., for simulating the reaction process of C-2 hydrogenation, obtaining the distributions of various physical parameters in the reactor, and by optimizing the operating conditions in the reaction process, the optimal inlet temperature and hydrogen/acetylene ratio are obtained; as a result, we can improve the ethylene selectivity and acetylene conversion rate. For example, Hu et al. (2015) [14] adopted a genetic algorithm for fitting the kinetic parameters of C-2 hydrogenation reaction, and the temperature distribution and ethylene concentration distribution in the reactor were obtained by using process simulation software Fluent. The optimal reaction temperature and hydrogen/acetylene ratio were obtained by maximizing the hydrogenation selectivity. Finally, the change of selectivity with inlet temperature and hydrogen/acetylene ratio was 
analyzed. The parameters of reaction kinetics model and inactivity kinetics model were fitted by using the genetic algorithm in the literature Tian et al. (2012) [15]. The selectivity and activity of two different catalysts were compared, and optimization methods such as combinatorial optimization and load distribution optimization were proposed to improve the selectivity and yield of ethylene. Wang et al. (2012) [16] obtained the kinetic equation of hydrogenation rate of ethylene and acetylene by using process simulation software Aspen Plus. According to the existing equations, the steady and dynamic models of the reactor were established by using process simulation software HYSYS, and the simulation results were in close agreement with the design data, which verified the accuracy of the model. Finally, step response characteristics of inlet temperature of the reactor and hydrogen/acetylene ratio of the feedstock were analyzed. Du et al. (2017) [17] studied mainly the operating conditions of the two-stage tandem acetylene hydrogenation reactor. When the catalyst was in the state of dynamic inactivation, the optimal operating conditions of the system were given by taking the ethylene selectivity and acetylene conversion rate as the optimization objective.

Through the above analysis, it can be seen that at present, for the modeling and optimization of C-2 hydrogenation reactors, there are more researches on isothermal reactors and relatively few researches on adiabatic reactors, and the current researches are focused on reactor mechanism model and process simulation, there has been few CFD investigation for the modeling and optimization of the adiabatic reactor.

In this paper, a C-2 hydrogenation adiabatic reactor was taken as the research object. On the basis of the established mathematical model of the adiabatic reaction process of C-2 hydrogenation, CFD was used to simulate the reaction process accurately, and the distribution of physical parameters in the reactor was obtained. By modifying the operating conditions of the reaction, a set of optimal temperature and hydrogen/acetylene ratio was obtained, improving the comprehensive performance of the ethylene selectivity and acetylene conversion rate, which has an important guiding role for the simulation and optimization of C-2 hydrogenation adiabatic reactor in industry.

\section{Materials and Methods}

In the adiabatic reaction of C-2 hydrogenation, there are solid and fluid phases in the porous medium. In order to simplify the problem and reduce the complexity of the model, a quasihomogeneous phase model is adopted, that is, the gas phase and the solid phase are regarded as unified phases without considering the mass exchange and energy exchange between them.

\subsection{Flow Model}

The middle section of the C-2 hydrogenation reactor is the main reaction zone, where both gaseous phase components and solid phase catalysts exist. It can be considered as a porous medium, which can be described by the porous medium model. The governing equations of the porous medium model mainly include mass conservation equation, momentum conservation equation, energy conservation equation and component mass conservation equation, which are respectively described as follows.

(1) Mass conservation equation

$$
\operatorname{div}\left(\varphi \rho_{g} \vec{v}\right)=0
$$

where, div is the divergence of the vector, $\varphi$ is the porosity, $\rho_{g}\left(\mathrm{~kg} / \mathrm{m}^{3}\right)$ is the gas phase density, and $\vec{v}(\mathrm{~m} / \mathrm{s})$ is the gas velocity. Since steady-state flow is simulated, the partial derivative of density with respect to time is ignored, and the same is done for the following conservation equations.

(2) Momentum conservation equation

$$
\operatorname{div}\left(\varphi \rho_{g} \vec{v} \vec{v}\right)=-\varphi \nabla p+\operatorname{div}(\varphi \vec{\tau})+\varphi \rho_{g} \vec{g}-\vec{S}
$$

where, $\vec{\tau}(\mathrm{Pa})$ is the stress-strain tensor, $\vec{g}\left(\mathrm{~m} / \mathrm{s}^{2}\right)$ is the gravitational volumetric force, and $\vec{S}$ is the momentum source term, which is given by the following formula: 


$$
\vec{S}=\frac{150 \mu}{D_{p}^{2}} \frac{(1-\varepsilon)^{2}}{\varepsilon^{3}} v+\frac{1.75 \rho(1-\varepsilon)}{D_{p} \varepsilon^{3}}|v| v
$$

where, $\mu$ (Pa s) is the viscosity, $D_{p}(\mathrm{~m})$ is the catalyst particle diameter, and $\varepsilon$ is the porosity.

(3) Energy conservation equation

The gas phase energy equation can be expressed as:

$$
\operatorname{div}\left(\varphi \vec{v}\left(\rho_{g} E_{g}+p\right)\right)=\varphi \operatorname{div}\left(k_{g} \nabla T_{g}-\sum_{i=1}^{N_{r}} h_{i} \vec{J}_{i}+\vec{\tau} \cdot \vec{v}\right)+h_{v}\left(T_{s}-T_{g}\right)
$$

The solid phase energy equation can be expressed as:

$$
0=\operatorname{div}\left((1-\varphi) k_{s} \nabla T_{s}\right)-h_{v}\left(T_{s}-T_{g}\right)
$$

where, $E_{g}(\mathrm{~J})$ is the gas phase energy, $k_{g}(\mathrm{~W} /(\mathrm{m} \mathrm{K}))$ is the thermal conductivity of gas phase, $k_{s}$ $(\mathrm{W} /(\mathrm{m} \mathrm{K}))$ is the thermal conductivity of solid phase, $T_{g}(\mathrm{~K})$ is the temperature of gas phase, $T_{s}$ $(\mathrm{K})$ is the temperature of solid phase, $h_{i}(\mathrm{~kJ} / \mathrm{kmol})$ is the species enthalpy of formation, and $h_{v}$ $\left(\mathrm{W} /\left(\mathrm{m}^{3} \mathrm{~K}\right)\right)$ is the gas-solid heat transfer coefficient.

For the C-2 hydrogenation reaction, we use a quasi-homogeneous model; hence, we just have to consider the energy equation of the gas phase, and the convective heat transfer between gas and solid phases can be ignored.

(4) Component mass conservation equation

$$
\frac{\partial}{\partial t}\left(\varphi \rho_{g} Y_{i}\right)+\nabla \cdot\left(\varphi \rho_{g} \vec{v} Y_{i}\right)=-\varphi \nabla \cdot \vec{J}_{i}+R_{i}+S_{i}
$$

where, $Y_{i}$ is the mass fraction of the component $i, \vec{J}_{i}\left(\mathrm{~kg} /\left(\mathrm{m}^{2} \mathrm{~s}\right)\right)$ is the diffusion flow density of the component $i, R_{i}\left(\mathrm{~kg} /\left(\mathrm{m}^{3} \mathrm{~s}\right)\right)$ is the net production rate of the chemical reaction, and $S_{i}$ $\left(\mathrm{kg} /\left(\mathrm{m}^{3} \mathrm{~s}\right)\right)$ is the additional production rate caused by the discrete phase and user-defined source term.

In equation (6), the diffusion flux of a substance is generated by the concentration gradient. Mass diffusion in laminar flow can be expressed as:

$$
\vec{J}_{i}=-\rho_{g} D_{i, m} \nabla Y_{i}
$$

where, $D_{i, m}\left(\mathrm{~m}^{2} / \mathrm{s}\right)$ is the diffusion coefficient of the ith substance in the mixture.

Mass diffusion in turbulence can be expressed as:

$$
\vec{J}_{i}=-\left(\rho_{g} D_{i, m}+\frac{\mu_{t}}{S c_{t}}\right) \nabla Y_{i}
$$

where, $\mu_{t}$ (Pa s) is the turbulent viscosity, and $S c_{t}$ is the turbulent Schmidt number, $S c_{t}=0.7$.

For the $\mathrm{C}-2$ hydrogenation reaction, the flow form in the porous medium region is laminar, so mass diffusion can be described by formula (7).

(5) Turbulence model

According to the research [1], the turbulence model of the adiabatic reactor uses the standard two $k-\varepsilon$ equations, which exhibit advantages of broad application, high precision, and appropriate computational cost.

The turbulent energy transport equation ( $k$ equation) can be expressed as: 


$$
\frac{\partial}{\partial t}\left(\rho_{g} k\right)+\frac{\partial}{\partial x_{i}}\left(\rho_{g} k u_{i}\right)=\frac{\partial}{\partial x_{j}}\left[\left(\mu_{g}+\frac{\mu_{t}}{\sigma_{k}}\right) \frac{\partial k}{\partial x_{j}}\right]+G_{k}-Y_{k}+S_{k}
$$

The energy dissipation transport equation ( $\varepsilon$ equation) can be expressed as:

$$
\frac{\partial}{\partial t}\left(\rho_{g} \varepsilon\right)+\frac{\partial}{\partial x_{i}}\left(\rho_{g} \varepsilon u_{i}\right)=\frac{\partial}{\partial x_{j}}\left[\left(\mu_{g}+\frac{\mu_{t}}{\sigma_{\varepsilon}}\right) \frac{\partial \varepsilon}{\partial x_{j}}\right]+C_{1 \varepsilon} \frac{\varepsilon}{k}\left(G_{k}+C_{3 \varepsilon} G_{b}\right)-C_{2 \varepsilon} \rho_{g} \frac{\varepsilon^{2}}{k}+S_{\varepsilon}
$$

where, $k\left(\mathrm{~m}^{2} / \mathrm{s}^{2}\right)$ is the turbulent kinetic energy, $\mu_{g}(\mathrm{~kg} /(\mathrm{m} \mathrm{s}))$ is the viscosity of gas molecules, $\mu_{t}\left((\mathrm{~kg} /(\mathrm{m} \mathrm{s}))\right.$ is the turbulent viscosity, $\sigma_{k}$ is the standard $k-\varepsilon$ model constant, $\sigma_{k}=1.0, G_{k}$ $\left(\mathrm{J} / \mathrm{m}^{3} / \mathrm{s}\right)$ is the generation of turbulent kinetic energy, $Y_{k}\left(\mathrm{~J} / \mathrm{m}^{3} / \mathrm{s}\right)$ is a fluctuation caused by transitional diffusion in compressible turbulence, $S_{k}\left(\mathrm{~J} / \mathrm{m}^{3} / \mathrm{s}\right)$ is user-defined, $\varepsilon\left(\mathrm{m}^{2} / \mathrm{s}^{3}\right)$ is the dissipation rate of turbulent kinetic energy, $\sigma_{\varepsilon}$ is the standard $k-\varepsilon$ model constant, $\sigma_{\varepsilon}=1.3$, $C_{1 \varepsilon}$ is the standard $k-\varepsilon$ model constant, $C_{1 \varepsilon}=1.44, C_{3 \varepsilon}$ is the standard $k-\varepsilon$ model constant, $G_{b}\left(\mathrm{~J} / \mathrm{m}^{3} / \mathrm{s}\right)$ is the turbulent kinetic energy generated by buoyancy, $C_{2 \varepsilon}$ is the standard $k-\varepsilon$ model constant, $C_{2 \varepsilon}=1.92$, and $S_{\varepsilon}\left(\mathrm{kg} /\left(\mathrm{m} \mathrm{s}^{4}\right)\right)$ is user-defined.

\subsection{Reaction Kinetic Model}

C-2 hydrogenation reaction refers to the process that acetylene is converted into ethylene or ethane by catalytic hydrogenation in the presence of the catalyst. Due to the different temperature, pressure, hydrogen/acetylene ratio and other conditions in the reaction process, in terms of the possibility of hydrogenation, the following two reactions may occur:

$$
\begin{aligned}
& \mathrm{C}_{2} \mathrm{H}_{2}+\mathrm{H}_{2} \rightarrow \mathrm{C}_{2} \mathrm{H}_{4}+174.3 \mathrm{~kJ} / \mathrm{mol} \\
& \mathrm{C}_{2} \mathrm{H}_{4}+\mathrm{H}_{2} \rightarrow \mathrm{C}_{2} \mathrm{H}_{6}+136.7 \mathrm{~kJ} / \mathrm{mol}
\end{aligned}
$$

According to the research [1], the reaction rate equations for the hydrogenation of acetylene and ethylene can be expressed as:

The kinetic equation of acetylene hydrogenation:

$$
r_{1}=1.129 \times 10^{13} \exp \left(-7.245 \times 10^{4} / R T\right)
$$

The kinetic equation of ethylene hydrogenation:

$$
r_{2}=2.228 \times 10^{13} \exp \left(-9.023 \times 10^{4} / R T\right)
$$

where, $r_{1}, r_{2}\left(\mathrm{kmol} / \mathrm{m}^{3} / \mathrm{s}\right)$ are the reaction rate, $R$ is the ideal gas constant, $R=8.314 \mathrm{~J} / \mathrm{mol} / \mathrm{K}$, and $T(\mathrm{~K})$ is the process gas temperature.

In this paper, the kinetic model of $\mathrm{C}-2$ hydrogenation reaction plans to adopt the kinetic equation given above, and then modify it to some extent according to the difference between the simulation results and the actual industrial data, so that it can be in close agreement with the actual industrial data.

\subsection{Grid Division}

Gambit, Fluent's pre-processing software, was used to divide the grid, which can be divided into three steps: first, establish the geometric model of the reactor; second, divide the grid of the reactor; third, define the boundary conditions of the reactor. 
The geometric model of the reactor is relatively simple. The middle section is a porous medium zone, the main reaction zone, which is composed of a cylinder with an inner diameter of $3500 \mathrm{~mm}$ and a height of $3316 \mathrm{~mm}$. The two ends are composed of hemispheres and do not react, and there exists only fluid flow and mixing.

For grid division, the element type is Tet/Hybrid, which is a tetrahedral Hybrid grid, and the division strategy is TGrid, which mainly uses tetrahedral elements, but hexahedral, pyramidal, wedge, and other elements can also be used at appropriate locations. Finally, 278,097 nodes and 1504,560 elements are determined, including 1492,278 tetrahedral elements, 12,282 pyramid elements, 3064,996 faces, 3 cell zones and 10 face zones.

The inlet is set as the mass flow inlet, the outlet as the pressure outlet, the wall as the wall type, and the intermediate reaction zone as the porous medium zone.

The geometric model of the reactor is shown in Figure 2, and the grid division is shown in Figure 3.

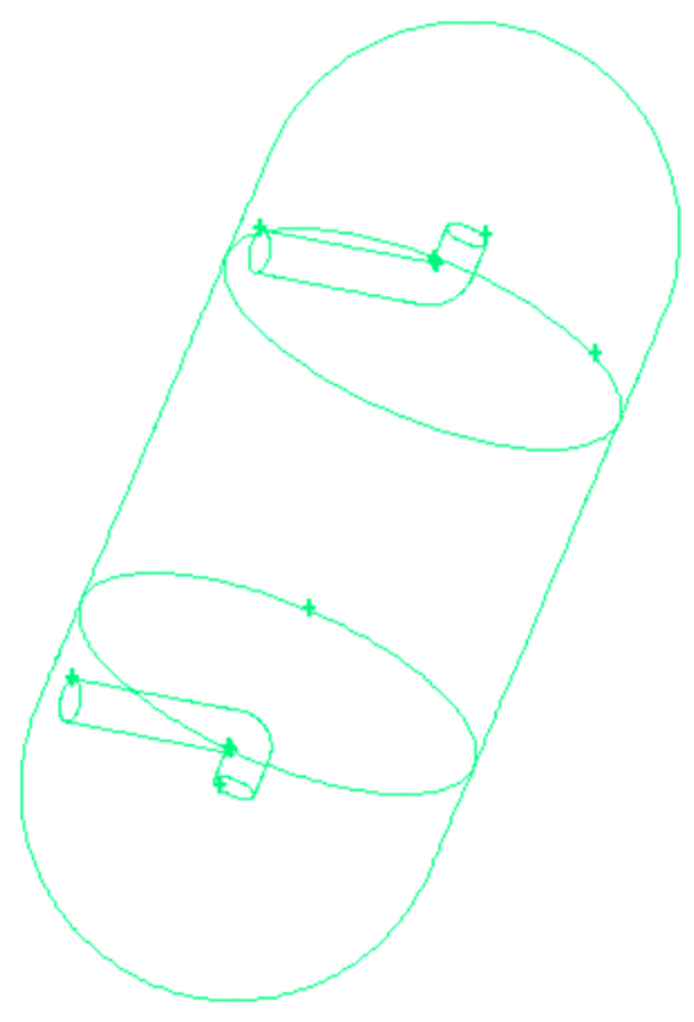

Figure 2. Geometric model of the reactor. 


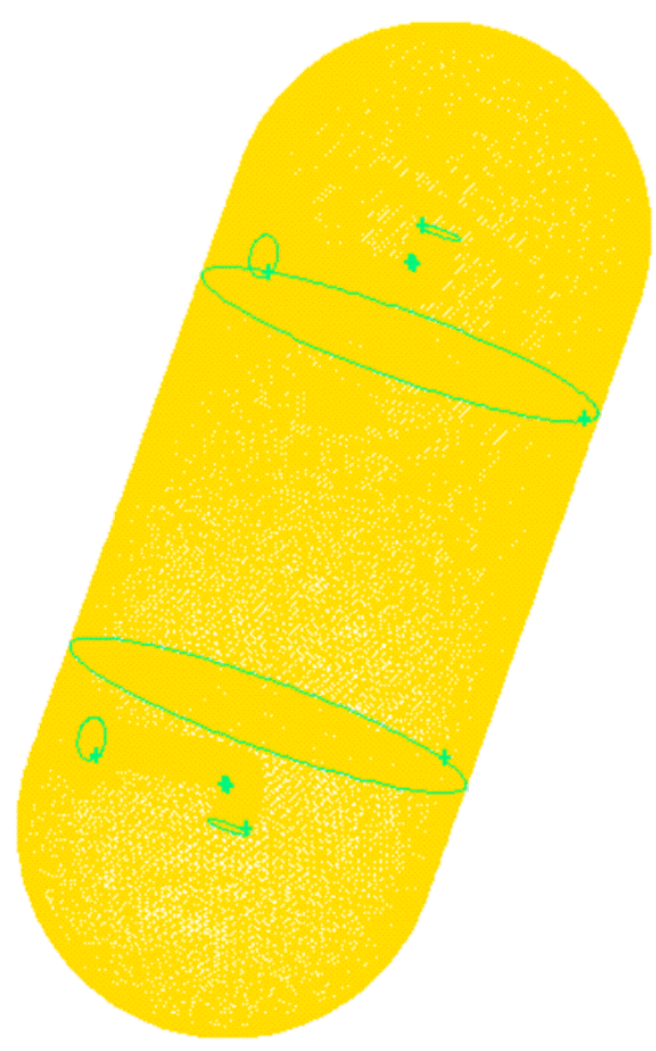

Figure 3. Grid division for the reactor.

\subsection{Boundary Conditions}

1. Inlet boundary condition

The inlet boundary conditions are determined as the mass flow inlet boundary, with the mass flow rate of $51.8721 \mathrm{~kg} / \mathrm{s}$, static pressure of $1.75 \mathrm{MPa}$, turbulence intensity of $2.1105 \%$, hydraulic diameter of $0.356 \mathrm{~m}$, total temperature of $320 \mathrm{~K}$, mass fraction of $\mathrm{C}_{2} \mathrm{H}_{6}, \mathrm{C}_{2} \mathrm{H}_{2}$ and $\mathrm{H}_{2}$ of $0.2204,0.0114$ and 0.00084148 respectively.

2. Outlet boundary condition

The outlet boundary conditions are determined as the pressure outlet boundary, the static pressure is $1.75 \mathrm{MPa}$, the backflow turbulence intensity is $2.1138 \%$, the backflow hydraulic diameter is $0.356 \mathrm{~m}$, and the total backflow temperature is determined as $300 \mathrm{~K}$.

3. Wall boundary conditions

The wall condition is determined as wall type, the shear condition is determined as the no-slip boundary condition, the roughness height and roughness constant use default values, and the heat flux is determined as 0 due to the reaction heat insulation.

\subsection{CFD Modeling Method}

The commercial software ANSYS Fluent 14.5 was employed for solving the governing equations of the porous medium model and the kinetic equation of hydrogenation reaction, and the finite volume method is adopted for obtaining the difference equations of governing equations. For C-2 hydrogenation reaction, finite rate chemical reaction model is used for the reaction model, the reaction type is Volumetric, and Laminar Finite-Rate model is used for the turbulence-chemistry interaction, in other words, the reaction velocity is calculated by the Arrhenius equation. SIMPLE algorithm is used for solving the coupled momentum, energy, and species transport equations. Gradient is discretized using Least Squares Cell Based scheme, Pressure is discretized using Standard scheme, and other physical parameters First Order Upwind scheme. Meanwhile, the 
under-relaxation factors for pressure, momentum, $k-\varepsilon$, and species are adjusted to lower values so that the simulation can converge. The default convergence criterion for the energy equation is $1 \mathrm{e}-$ 06, and the convergence criteria for other equations are 0.001 .

\subsection{Model Parameters}

The model parameters mainly include the physical parameters of components and the physical parameters of the gas mixture, as shown in Table 1 and Table 2.

Table 1. Component physical parameters.

\begin{tabular}{cccccc}
\hline Species & $\begin{array}{c}C_{p} \\
(\mathbf{J} / \mathbf{k g}-\mathbf{K})\end{array}$ & $\begin{array}{c}M \\
(\mathbf{k g} / \mathbf{k m o l})\end{array}$ & $\begin{array}{c}H \\
(\mathbf{J} / \mathbf{k m o l})\end{array}$ & $\begin{array}{c}S \\
(\mathbf{J} / \mathbf{k m o l}-\mathbf{K})\end{array}$ & $\begin{array}{c}T \\
(\mathbf{K})\end{array}$ \\
\hline $\mathrm{H}_{2}$ & piecewise-polynomial & 2.01594 & 0 & 130579.1 & 298.15 \\
$\mathrm{C}_{2} \mathrm{H}_{2}$ & piecewise-polynomial & 26.04 & $2.26751 \mathrm{e}+08$ & 200892.9 & 298.15 \\
$\mathrm{C}_{2} \mathrm{H}_{4}$ & piecewise-polynomial & 28.05418 & $5.24554 \mathrm{e}+07$ & 219148.6 & 298.15 \\
$\mathrm{C}_{2} \mathrm{H}_{6}$ & piecewise-polynomial & 30.07012 & $8.386329 \mathrm{e}+07$ & 228949.3 & 298.15 \\
\hline
\end{tabular}

Table 2. Mixture physical parameters.

\begin{tabular}{cccccc}
\hline Species & $\begin{array}{c}C_{p, g} \\
(\mathbf{J} / \mathbf{k g}-\mathbf{K})\end{array}$ & $\begin{array}{c}\rho_{g} \\
(\mathbf{k g} / \mathbf{m} 3)\end{array}$ & $\begin{array}{c}k_{g} \\
(\mathbf{W} / \mathbf{m}-\mathbf{K})\end{array}$ & $\begin{array}{c}\mu_{g} \\
(\mathbf{k g} / \mathbf{m}-\mathbf{s})\end{array}$ & $\begin{array}{c}D_{m} \\
(\mathbf{m} 2 / \mathbf{s})\end{array}$ \\
\hline mixture & mixing-law & ideal-gas & 0.0454 & $1.72 \mathrm{e}-05$ & $2.88 \mathrm{e}-05$ \\
\hline
\end{tabular}

\section{Results and Discussion}

\subsection{Convergence Analysis}

The inlet and outlet mass flow report are shown in Figure 4. It can be seen from the figure that the inlet mass flow is $51.8721 \mathrm{~kg} / \mathrm{s}$, the outlet mass flow is $-51.836859 \mathrm{~kg} / \mathrm{s}$, and the net mass flow is $0.03524108 \mathrm{~kg} / \mathrm{s}$. The inlet and outlet mass flow are not exactly equal, but the relative error is $0.0679 \%$ that is less than $0.1 \%$, which is within the acceptable range and can be considered to meet the conservation of mass. 


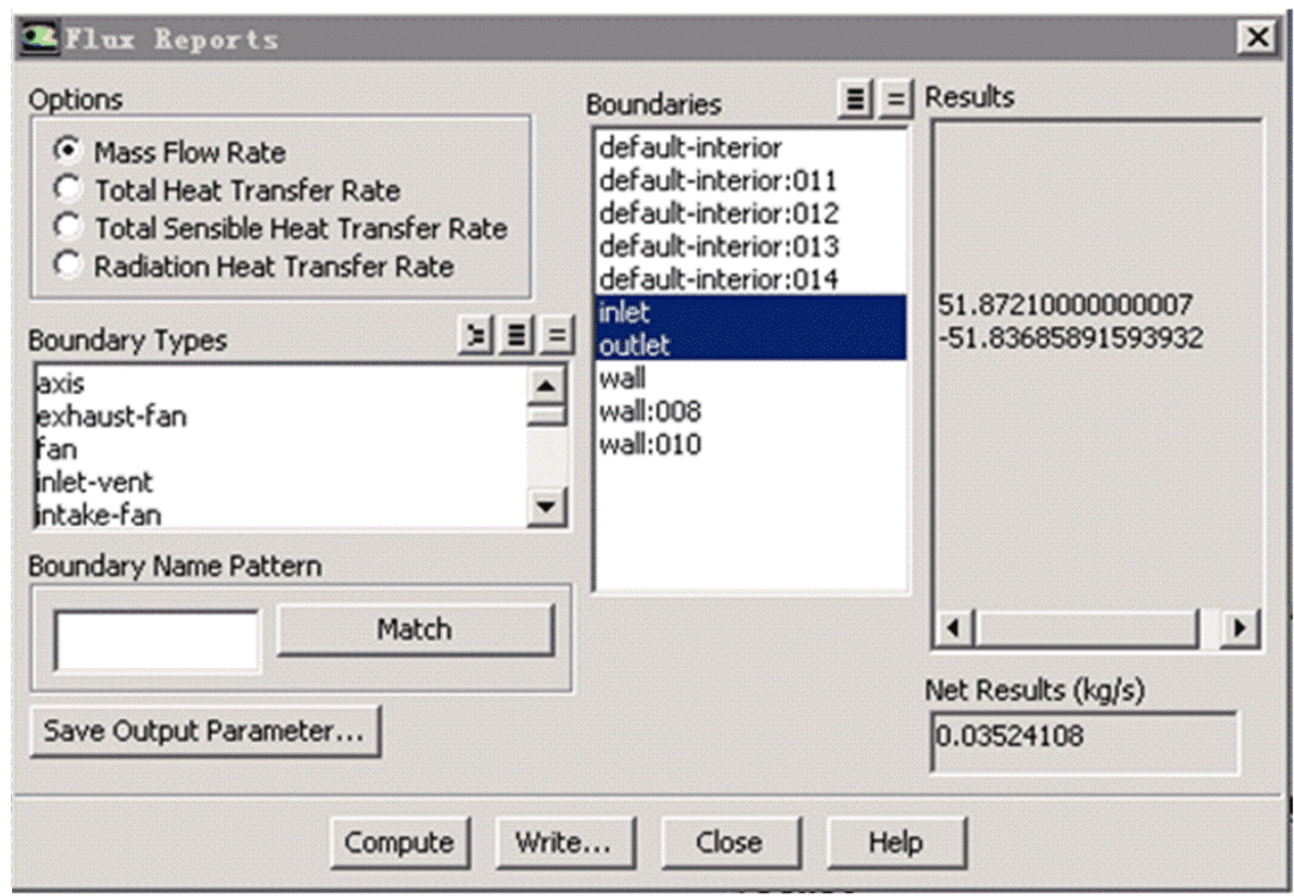

Figure 4. Inlet and outlet mass flow.

The inlet and outlet heat transfer rate are shown in Figure 5. It can be seen from the figure that the inlet heat transfer rate is $49534251 \mathrm{~W}$, the outlet heat transfer rate is $-49531142 \mathrm{~W}$, and the net heat transfer rate is $3,108.816 \mathrm{~W}$. The relative error is $0.0063 \%$ that is less than $0.1 \%$, which is within the acceptable range and meets the energy conservation.

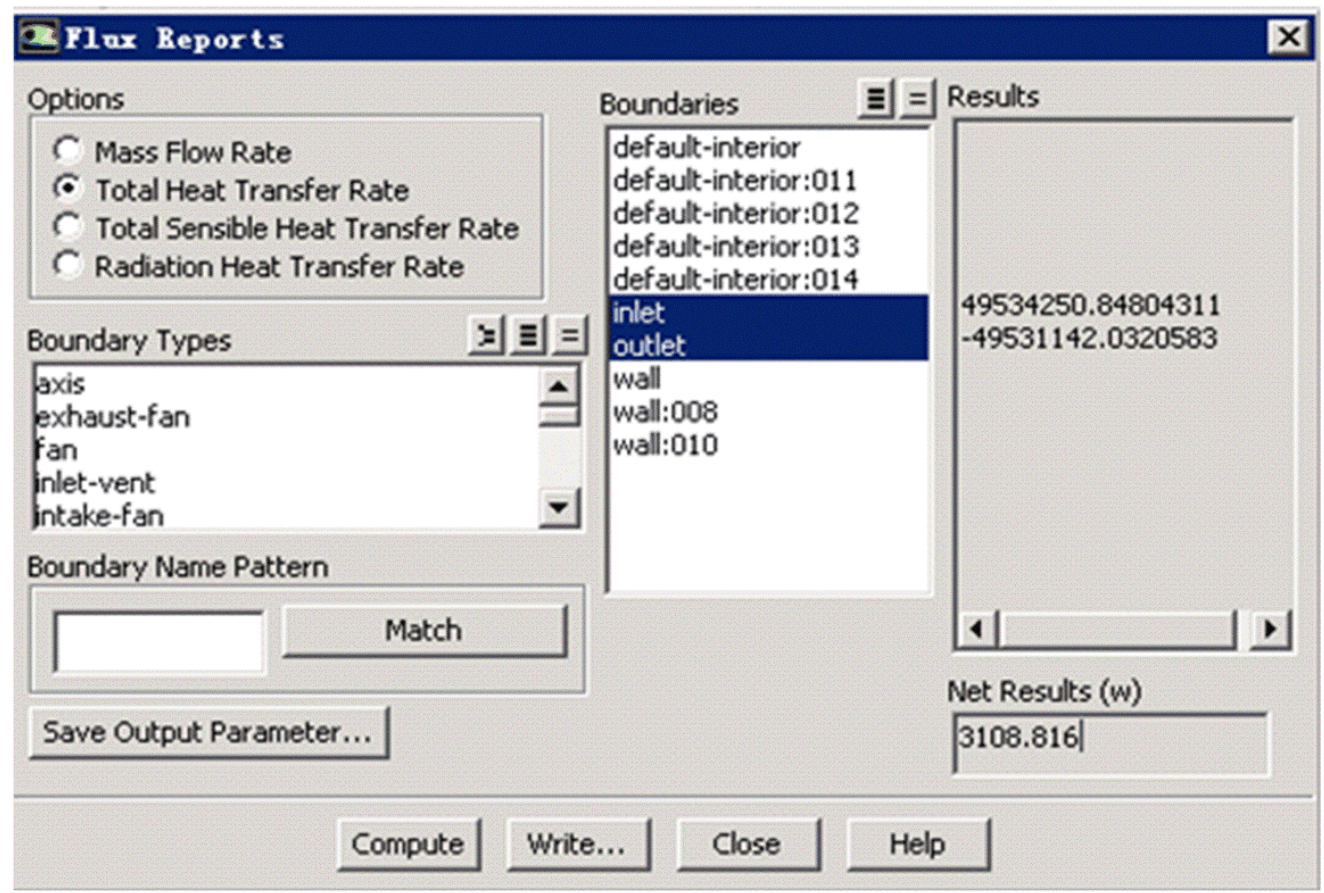

Figure 5. Inlet and outlet heat transfer rate.

From the flux reports, it can be seen that the calculation results meet the conservation of mass and energy, which can be considered as convergent and reliable.

Figure 6 shows the convergence curve of outlet mass flow. It can be seen from the figure that after about 1000 iterations, the outlet mass flow is $-51.8369 \mathrm{~kg} / \mathrm{s}$, which is close to the inlet flow and the curve tends to be stable. It can be considered that the calculation results are reliable and the calculation has converged. 


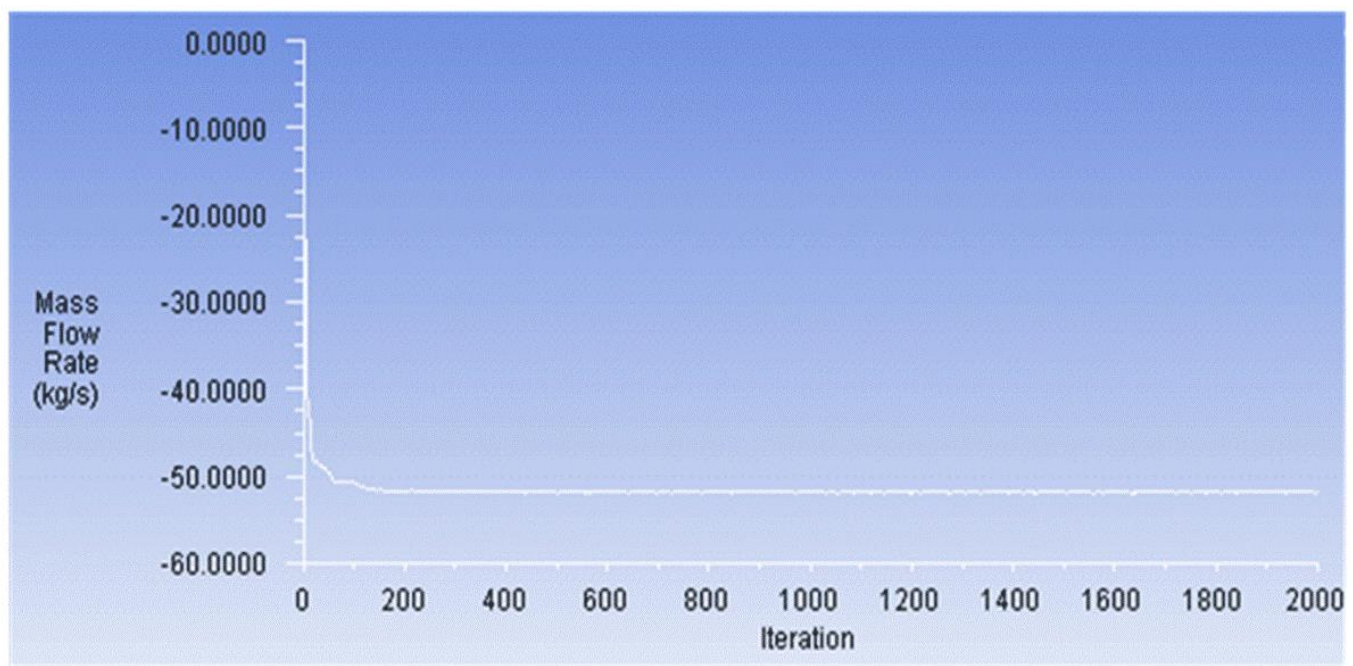

Figure 6. Convergence curve for the outlet mass flow.

\subsection{Model Validation}

The kinetic parameters of each section used in the simulation are shown in Table 3 , where, $A_{1}$ and $E_{1}$ are the kinetic parameters of acetylene hydrogenation, and $A_{2}$ and $E_{2}$ are the kinetic parameters of ethylene hydrogenation.

Table 3. The kinetic parameters of the reactor.

\begin{tabular}{ccccc}
\hline Kinetic parameters & \multirow{2}{*}{$A_{1}$} & $\begin{array}{c}E_{1} \\
(\mathbf{J} / \mathbf{K m o l})\end{array}$ & $A_{2}$ & $\begin{array}{c}E_{2} \\
\mathbf{( J / k m o l})\end{array}$ \\
\hline First section & $1.129 \mathrm{e}+13$ & $7.245 \mathrm{e}+07$ & $2.228 \mathrm{e}+13$ & $9.0227 \mathrm{e}+07$ \\
Second section & $6.2853 \mathrm{e}+13$ & $7.245 \mathrm{e}+07$ & $2.228 \mathrm{e}+13$ & $9.0227 \mathrm{e}+07$ \\
Third section & $1.1129 \mathrm{e}+14$ & $7.245 \mathrm{e}+07$ & $2.228 \mathrm{e}+13$ & $9.0227 \mathrm{e}+07$ \\
\hline
\end{tabular}

The operating conditions used in the simulation are shown in Table 4.

Table 4. The inlet actual data.

\begin{tabular}{cccc}
\hline Items & First Section & Second Section & Third Section \\
\hline Inlet temperature/K & 320 & 324 & 337 \\
Inlet pressure/MPa & 1.75 & 1.75 & 1.75 \\
Inlet mass flow $/ \mathrm{kg} / \mathrm{s}$ & 51.8721 & 51.89 & 51.91 \\
Inlet mass fraction of $\mathrm{C}_{2} \mathrm{H}_{6} / \mathrm{wt} \%$ & 22.04 & 22.47 & 22.87 \\
Inlet mass fraction of $\mathrm{C}_{2} \mathrm{H}_{4} / \mathrm{wt} \%$ & 76.74 & 76.98 & 77.10 \\
Inlet mass fraction of $\mathrm{C}_{2} \mathrm{H}_{2} / \mathrm{wt} \%$ & 1.14 & 0.48 & $6.55 \mathrm{e}-03$ \\
Inlet mass fraction of $\mathrm{H}_{2} / \mathrm{wt} \%$ & $8.41 \mathrm{e}-02$ & $6.01 \mathrm{e}-02$ & $2.08 \mathrm{e}-02$ \\
\hline
\end{tabular}

The structure of the reactor and operating conditions used in the simulation were consistent with the actual data, and the simulation results were shown in Table 5-7.

Table 5. Comparison between simulation results and actual data in the first section. 


\begin{tabular}{cccc}
\hline Outlet temperature $/ \mathrm{K}$ & 351 & 353 & 0.57 \\
Intermediate temperature point $T_{1} / \mathrm{K}$ & 331 & 331 & 0.00 \\
Intermediate temperature point $\mathrm{T}_{2} / \mathrm{K}$ & 343 & 345 & 0.58 \\
Intermediate temperature point $\mathrm{T}_{3} / \mathrm{K}$ & 349 & 349 & 0.00 \\
Outlet pressure $/ \mathrm{MPa}$ & 1.75 & 1.75 & 0.00 \\
Outlet mass flow $/ \mathrm{kg} / \mathrm{s}$ & 51.8721 & 51.8369 & 0.07 \\
Outlet mass fraction of $\mathrm{C}_{2} \mathrm{H}_{6} / \mathrm{wt} \%$ & 22.48 & 22.11 & 1.65 \\
Outlet mass fraction of $\mathrm{C}_{2} \mathrm{H}_{4} / \mathrm{wt} \%$ & 77.00 & 77.51 & 0.66 \\
Outlet mass fraction of $\mathrm{C}_{2} \mathrm{H}_{2} / \mathrm{wt} \%$ & 0.40 & 0.38 & 5.00 \\
\hline
\end{tabular}

Table 6. Comparison between simulation results and actual data in the second section.

\begin{tabular}{cccc}
\hline Items & Actual Data & Simulation Results & Error\% \\
\hline Outlet temperature $/ \mathrm{K}$ & 348 & 350 & 0.57 \\
Intermediate temperature point $\mathrm{T}_{1} / \mathrm{K}$ & 340 & 340 & 0.00 \\
Intermediate temperature point $\mathrm{T}_{2} / \mathrm{K}$ & 345 & 345 & 0.00 \\
Intermediate temperature point $\mathrm{T}_{3} / \mathrm{K}$ & 347 & 348 & 0.29 \\
Outlet pressure/MPa & 1.75 & 1.75 & 0.00 \\
Outlet mass flow $/ \mathrm{kg} / \mathrm{s}$ & 51.89 & 51.86 & 0.06 \\
Outlet mass fraction of $\mathrm{C}_{2} \mathrm{H}_{6} / \mathrm{wt} \%$ & 22.87 & 22.45 & 1.84 \\
Outlet mass fraction of $\mathrm{C}_{2} \mathrm{H}_{4} / \mathrm{wt} \%$ & 77.11 & 77.54 & 0.56 \\
Outlet mass fraction of $\mathrm{C}_{2} \mathrm{H}_{2} / \mathrm{wt} \%$ & $6.55 \mathrm{e}-03$ & $6.60 \mathrm{e}-03$ & 0.76 \\
\hline
\end{tabular}

Table 7. Comparison between simulation results and actual data in the third section.

\begin{tabular}{cccc}
\hline Items & Actual Data & Simulation Results & Error\% \\
\hline Outlet temperature/K & 346 & 348 & 0.58 \\
Intermediate temperature point $\mathrm{T}_{1} / \mathrm{K}$ & 342 & 342 & 0.00 \\
Intermediate temperature point $\mathrm{T}_{2} / \mathrm{K}$ & 345 & 344 & 0.29 \\
Intermediate temperature point $\mathrm{T}_{3} / \mathrm{K}$ & 346 & 346 & 0.00 \\
Outlet pressure/MPa & 1.75 & 1.75 & 0.00 \\
Outlet mass flow $/ \mathrm{kg} / \mathrm{s}$ & 51.91 & 51.88 & 0.06 \\
Outlet mass fraction of $\mathrm{C}_{2} \mathrm{H}_{6} / \mathrm{wt} \%$ & 23.17 & 23.76 & 2.55 \\
Outlet mass fraction of $\mathrm{C}_{2} \mathrm{H}_{4} / \mathrm{wt} \%$ & 76.83 & 76.23 & 0.78 \\
Outlet mass fraction of $\mathrm{C}_{2} \mathrm{H}_{2} / \mathrm{wt} \%$ & 0.00 & $4.67 \mathrm{e}-07$ & 0.58 \\
\hline
\end{tabular}

As can be seen from Table 5-7, the simulated outlet temperature and the temperature at intermediate temperature point are very close to the actual data. As the pressure outlet boundary condition is adopted, the outlet pressure and the inlet pressure are equal. The simulated outlet mass flow is close to the actual outlet mass flow, and the outlet mass fractions of $\mathrm{C}_{2} \mathrm{H}_{6}, \mathrm{C}_{2} \mathrm{H}_{4}$ and $\mathrm{C}_{2} \mathrm{H}_{2}$ are close to the actual outlet mass fraction. The relative errors of the critical parameters in the table are within $5 \%$. In general, the physical parameters of the simulation results, such as temperature, pressure, flow rate and mass fraction, are close to the actual data, which verifies the accuracy of the model and lays a solid foundation for the optimization of the following operating conditions.

\subsection{Flow Field Distribution}

Figure 7(a) shows the temperature distribution contours in the reactor. It can be seen from the figure that the temperature increases gradually from the mass flow inlet to the pressure outlet, the temperature is $320 \mathrm{~K}$ at the inlet, while $358 \mathrm{~K}$ at the outlet. However, at both ends of the reactor, the temperature basically remains unchanged, while in the porous medium region in the middle of the reactor, the temperature increases significantly. Theoretical analysis is as follows: C-2 hydrogenation reaction is an exothermic reaction and the reaction is adiabatic, so as the reaction progresses, temperature gradually rises along the direction of the gas flow, but at both ends of the reactor there exists only gas flow and mixing, there is no reaction; therefore, the temperature keeps unchanged. In 
the middle region of the reactor, temperature rises rapidly because of the large amount of heat given off by the reaction.

The pressure distribution contour in the reactor is shown in Figure 7(b), which shows that the pressure gradually decreases from the mass flow inlet to the pressure outlet. Similar to Figure 7(a), at both ends of the reactor, the pressure is basically unchanged, but in the porous medium region in the middle of the reactor, the pressure rapidly decreases. Theoretical analysis is as follows: there exists no reaction at both ends of the reactor, so the pressure is basically unchanged. However, in the porous medium region in the middle of the reactor, C-2 hydrogenation reaction occurs and it is a reaction in which the number of moles decreases. According to the ideal gas state equation, the reduction in the number of moles will lead to a reduction in the pressure, so the pressure will drop rapidly. It can be seen from the pressure contours that, although the pressure decreases from the inlet to the outlet, the reduced amount is very small, so it can be considered that the pressure basically has no change.

The velocity distribution contour in the reactor is shown in Figure 7(c). It can be seen from the figure that at the mass flow inlet, the velocity is relatively large, reaching up to $26.12 \mathrm{~m} / \mathrm{s}$. Then the process gas enters the middle porous medium region, under the action of viscous resistance and inertial resistance, the velocity gradually decreases to $0-2.23 \mathrm{~m} / \mathrm{s}$; when the process gas reaches the pressure outlet, the velocity rapidly increases to $42.01 \mathrm{~m} / \mathrm{s}$. Theoretical analysis is as follows: the cross-sectional area at the outlet is very small, according to the continuity equation, when the flow rate is constant, the decrease of cross-sectional area will lead to the increase of velocity. A similar analysis can be performed for the velocity at the inlet.

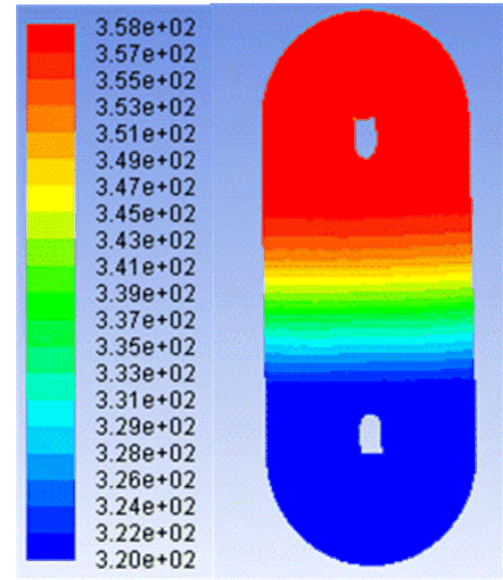

(a)

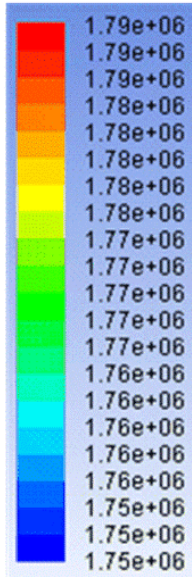

$1.75 e+06$

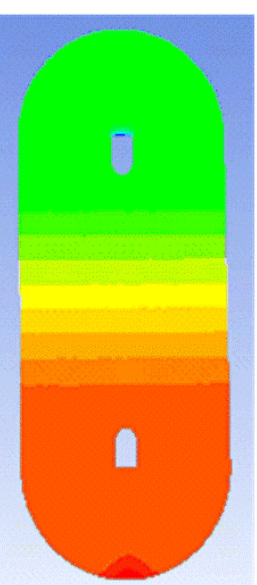

(b)

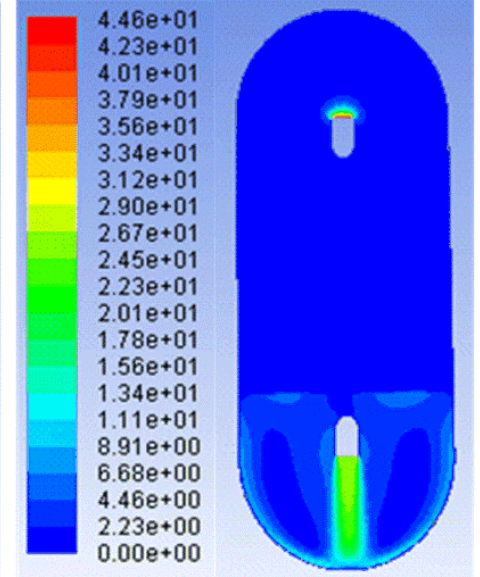

(c)

Figure 7. Physical field contours in the reactor: (a) Temperature field contours; (b) Pressure field contours; (c) Velocity field contours.

The contour of the mole fraction distribution of each component in the gas phase is shown in Figure 8. From the figure, the mole fractions of $\mathrm{H}_{2}$ and $\mathrm{C}_{2} \mathrm{H}_{2}$ decrease gradually from the mass flow inlet to the pressure outlet, while the mole fractions of $\mathrm{C}_{2} \mathrm{H}_{4}$ and $\mathrm{C}_{2} \mathrm{H}_{6}$ increase gradually. At both ends of the reactor, the mole fraction of each component is basically unchanged, while in the porous medium region in the middle of the reactor, the reaction is the most intense, which shows that the mole fraction of each component changes most obviously. According to the mechanism of C-2 hydrogenation reaction, in the middle of the reactor, there are two reactions of acetylene hydrogenation to ethylene and ethylene hydrogenation to ethane at the same time. In the first half of the reaction, acetylene concentration is relatively high, and acetylene hydrogenation reaction is strong and plays a leading role, which is manifested as the continuous decrease of acetylene concentration and the continuous increase of ethylene concentration. As the reaction progresses, acetylene concentration gradually reduces to a low value, while the concentration of ethylene is very large, ethylene hydrogenation reaction plays a leading role, showing the continuous increase of ethane concentration and the continuous decrease of hydrogen concentration; but the adsorption 
capacity of catalyst to ethylene molecule is very weak, the reaction is not strong, so the ethylene concentration doesn't decrease.

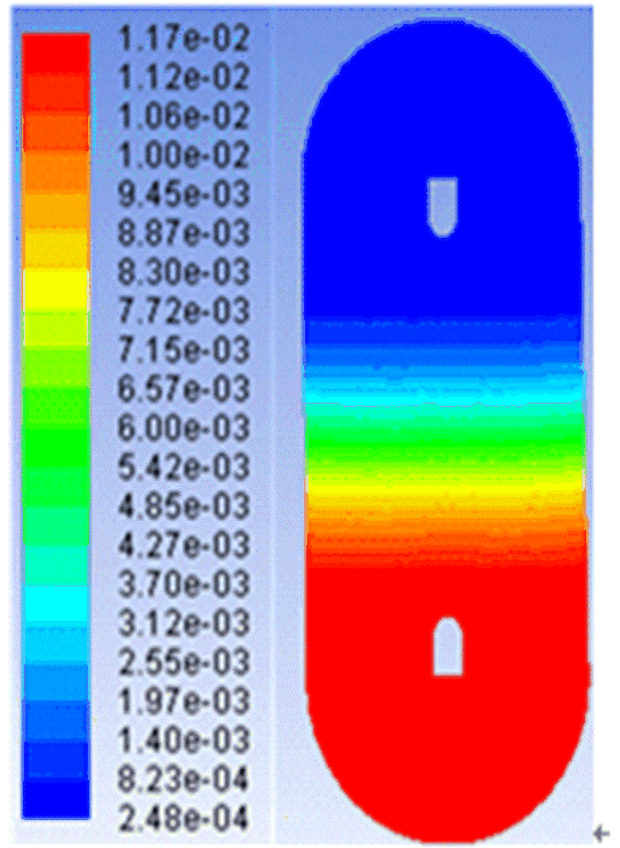

(a)

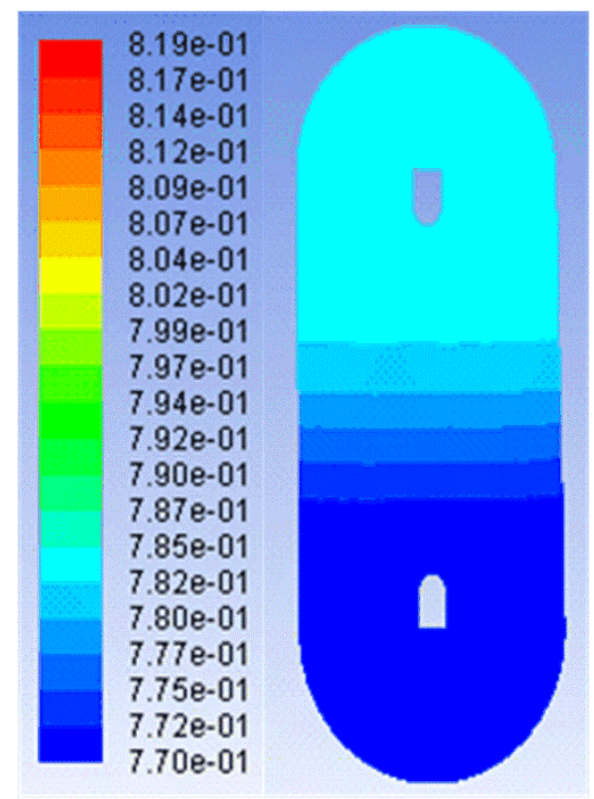

(c)

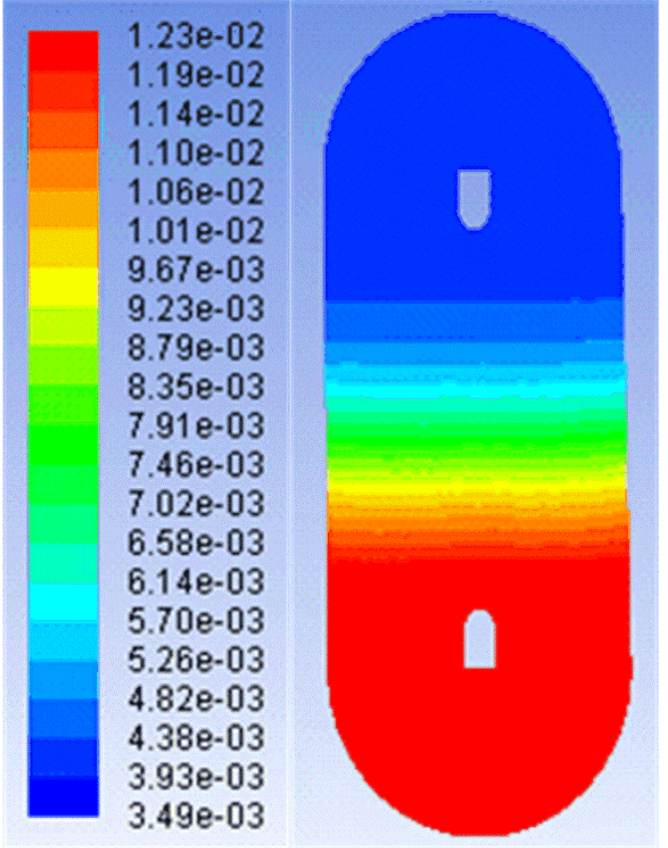

(b)

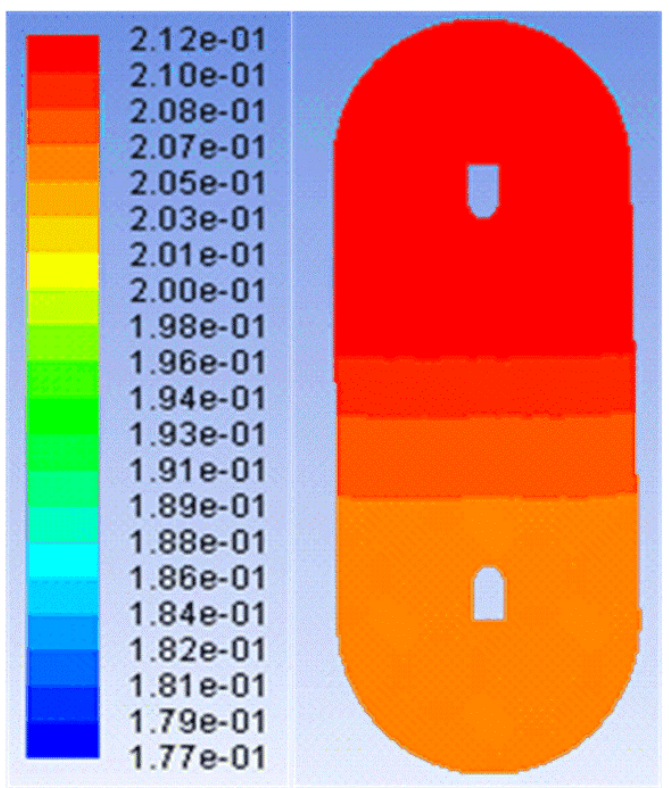

(d)

Figure 8. Component mole fraction contours: (a) $\mathrm{H}_{2}$ mole fraction contours; (b) $\mathrm{C}_{2} \mathrm{H}_{2}$ mole fraction contours; (c) $\mathrm{C}_{2} \mathrm{H}_{4}$ mole fraction contours; (d) $\mathrm{C}_{2} \mathrm{H}_{6}$ mole fraction contours.

\subsection{Optimization Study}

On the basis of the CFD simulation of C-2 hydrogenation adiabatic reaction process, the parameters in the reaction process, such as the reactor structure, inlet temperature, hydrogen/acetylene ratio etc., were optimized to obtain the optimal structural parameters, inlet temperature and hydrogen/acetylene ratio; as a result, we can improve the comprehensive performance of ethylene selectivity and acetylene conversion rate, and achieve maximum economic benefits under the premise that the outlet acetylene concentration meets the requirement. The 
operating conditions used in the simulation derived from the actual inlet data in Table 4 in Section 3.2.

\subsubsection{Structure Optimization}

The porous medium region in the middle of the reactor is the main reaction region. The volume of the porous medium region was kept unchanged, and the structure of the reactor was optimized by changing the diameter/height ratio; hence, we can achieve the optimal selectivity and conversion rate. We selected 11 sets of data, as shown in Table 8.

Table 8. Structural dimensions of the reactor.

\begin{tabular}{cccc}
\hline No. & D/m & L/m & D/L \\
\hline 1 & 3.0 & 4.513 & 0.6647 \\
2 & 3.1 & 4.227 & 0.7334 \\
3 & 3.2 & 3.967 & 0.8067 \\
4 & 3.3 & 3.730 & 0.8847 \\
5 & 3.4 & 3.514 & 0.9676 \\
6 & 3.5 & 3.316 & 1.0555 \\
7 & 3.6 & 3.134 & 1.1487 \\
8 & 3.7 & 2.967 & 1.2471 \\
9 & 3.8 & 2.813 & 1.3509 \\
10 & 3.9 & 2.671 & 1.4601 \\
11 & 4.0 & 2.539 & 1.5754 \\
\hline
\end{tabular}

According to literature [18], ethylene selectivity is defined as:

$$
S=\frac{x_{\mathrm{C}_{2} \mathrm{H} 2 \text {,in }}-x_{\mathrm{C}_{2} \mathrm{H} 2, \text { out }}}{x_{\mathrm{H}_{2, \text { in }}}-x_{\mathrm{H}_{2, \text { out }}}}
$$

where, $S$ represents the selectivity, $x_{\mathrm{C}_{2} \mathrm{H}_{2}, \text { in }}$ represents the inlet mole fraction of $\mathrm{C}_{2} \mathrm{H}_{2}, x_{\mathrm{C}_{2} \mathrm{H}_{2}, \text { out }}$ represents the outlet mole fraction of $\mathrm{C}_{2} \mathrm{H}_{2}, x_{\mathrm{H}_{2} \text {,in }}$ represents the inlet mole fraction of $\mathrm{H}_{2}$, and $x_{\mathrm{H}_{2} \text {,out }}$ represents the outlet mole fraction of $\mathrm{H}_{2}$. The physical meaning of Equation (15) is that the ratio of hydrogen reacted by acetylene hydrogenation to the total hydrogen reacted.

The acetylene conversion rate is defined as:

$$
C=\frac{x_{\mathrm{C}_{2} \mathrm{H} 2, \text { in }}-x_{\mathrm{C}_{2} \mathrm{H} 2, \text { out }}}{x_{\mathrm{C}_{2} \mathrm{H}_{2} \text { in }}}
$$

where, $C$ represents the conversion rate.

Under some conditions, ethylene selectivity and acetylene conversion rate are often contradictory, that is, when the selectivity is very high, the conversion rate is often very low; Iikewise, when the conversion rate is very high, the selectivity is often very low. Ethylene selectivity and acetylene conversion rate cannot both get the optimal value under the same operation parameter. In order to reconcile the contradiction between the ethylene selectivity and acetylene conversion rate, a third evaluation index was introduced, which was named $F 1$, and it was the harmonic mean of $S$ and $C$, namely:

$$
F 1=\frac{2 S C}{S+C}
$$

where, $F 1$ represents the harmonic mean of $S$ and $C$, which reflects the comprehensive performance metrics of hydrogenation reaction. The larger $F 1$ is, the better the comprehensive performance of selectivity and conversion rate will be. 
The ethylene selectivity, acetylene conversion rate and harmonic mean under different $D / L$ were obtained by changing the structure size of the reactor with the inlet condition of the reactor unchanged. The optimization results are shown in Table 9.

Table 9. The optimizing results under different $D / L$.

\begin{tabular}{cccc}
\hline$D / L$ & $S$ & $C$ & $F 1$ \\
\hline 0.6647 & 0.7063 & 0.6633 & 0.6841 \\
0.7334 & 0.7067 & 0.6631 & 0.6842 \\
0.8067 & 0.707 & 0.6627 & 0.6841 \\
0.8847 & 0.7076 & 0.6625 & 0.6843 \\
0.9676 & 0.7079 & 0.6623 & 0.6843 \\
1.0555 & 0.7083 & 0.6624 & 0.6846 \\
1.1487 & 0.7084 & 0.6624 & 0.6846 \\
1.2471 & 0.7089 & 0.6623 & 0.6848 \\
1.3509 & 0.7089 & 0.6623 & 0.6848 \\
1.4601 & 0.7092 & 0.6624 & 0.6850 \\
1.5754 & 0.7093 & 0.6625 & 0.6851 \\
\hline
\end{tabular}

It can be seen from Table 9 that, under the same inlet conditions, only changing $D / L$ has no significant influence on ethylene selectivity, acetylene conversion rate and harmonic mean; hence, it can be considered that changing the diameter/height ratio has no significant improvement on selectivity and conversion rate. Theoretical analysis is as follows: from the perspective of chemical kinetics, the factors affecting the chemical reaction rate mainly include the concentration of reactants, pressure, temperature and the activity of the catalyst. For C-2 hydrogenation reaction, the catalyst used in the reaction is the same, the pressure of the reactant is proportional to the concentration, and the feed amount of the reactant is constant, the volume of the reactor is also a constant; hence, the concentration of the reactant is also a constant; thus, the reaction rate is only related to the reaction temperature. If the temperature is the same, the reaction rate is also a fixed value; thus, the reaction rate is independent of $D / L$, and the outlet concentration of each component is basically unchanged; hence, the geometric size of the reactor exerts a marginal effect on selectivity, conversion rate and harmonic mean.

Temperature distribution under different $D / L$ is shown in Table 10 . The figure shows that, under the different $D / L$, the intermediate temperature point and outlet temperature of the reactor change marginally. It can be seen from the preceding analysis that, under the condition that the reaction temperature changes marginally, the reaction rate changes marginally, and the outlet concentration of each component changes marginally; hence, the corresponding selectivity, conversion rate and harmonic mean change marginally.

Table 10. The temperature distribution under different $D / L$. 


\begin{tabular}{ccccc}
\hline D/L & $\begin{array}{c}\text { Intermediate } \\
\text { Temperature Point } \\
\boldsymbol{T}_{\mathbf{1}} / \mathbf{K}\end{array}$ & $\begin{array}{c}\text { Intermediate } \\
\text { Temperature Point } \\
\mathbf{T}_{\mathbf{2}} / \mathbf{K}\end{array}$ & $\begin{array}{c}\text { Intermediate } \\
\text { Temperature Point } \\
\boldsymbol{T}_{\mathbf{3}} / \mathbf{K}\end{array}$ & $\begin{array}{c}\text { Outlet } \\
\text { Temperature }\end{array}$ \\
\hline 0.6647 & 331 & 346 & 356 & 359 \\
0.7334 & 331 & 345 & 356 & 359 \\
0.8067 & 331 & 345 & 355 & 358 \\
0.8847 & 331 & 345 & 355 & 358 \\
0.9676 & 331 & 345 & 355 & 358 \\
1.0555 & 331 & 345 & 355 & 358 \\
1.1487 & 331 & 344 & 355 & 358 \\
1.2471 & 331 & 344 & 355 & 358 \\
1.3509 & 331 & 344 & 355 & 358 \\
1.4601 & 331 & 344 & 355 & 358 \\
1.5754 & 331 & 344 & 355 & 358 \\
\hline
\end{tabular}

\subsubsection{Optimization of Operation Conditions}

The actual industrial inlet temperature in the C-2 hydrogenation reaction is $320 \mathrm{~K}$, and the hydrogen/acetylene ratio (mass fraction ratio) is 0.07. For the inlet temperature, we took 10 levels: 310 K, 312 K, 314 K, 316 K, 318 K, 320 K (before optimization), 322 K, 324 K, 326 K, 328 K. For hydrogen/acetylene ratio, we took 13 levels: $0.02,0.03,0.04,0.05,0.06,0.07$ (before optimization), 0.08, $0.09,0.10,0.11,0.12,0.13,0.14$.

If the comprehensive test method is adopted, a total of 130 tests will be performed. When enough factors and levels are taken into account, the number of tests will be too large to be realized. The single factor test rotation method was adopted to carry out iterative optimization analysis for the inlet temperature and hydrogen/acetylene ratio, and the optimal parameter can be found through only 46 tests.

In the first step, we fixed the hydrogen/acetylene ratio $R$ at 0.07 to optimize $T$, and obtained the ethylene selectivity, acetylene conversion rate and harmonic mean at different temperatures with other operating conditions unchanged, which were shown in Table 11.

Table 11. The optimization results of the first step.

\begin{tabular}{cccc}
\hline$T / K$ & $S$ & $C$ & $F 1$ \\
\hline 310 & 0.8226 & 0.4146 & 0.5513 \\
312 & 0.7895 & 0.4878 & 0.603 \\
314 & 0.7667 & 0.561 & 0.6479 \\
316 & 0.7451 & 0.6179 & 0.6756 \\
318 & 0.724 & 0.6504 & 0.6852 \\
320 & 0.7043 & 0.6585 & 0.6806 \\
322 & 0.7009 & 0.6667 & 0.6834 \\
324 & 0.6882 & 0.6585 & 0.673 \\
326 & 0.6869 & 0.6585 & 0.6724 \\
328 & 0.6781 & 0.6504 & 0.664 \\
\hline
\end{tabular}

As can be seen from Table 11, when the temperature is $318 \mathrm{~K}, F 1$ reaches a maximum value of 0.6852 ; therefore, the optimal parameter combination is: temperature $318 \mathrm{~K}$, hydrogen/acetylene ratio 0.07 .

In the second step, we fixed temperature $T$ at $318 \mathrm{~K}$ to optimize the hydrogen/acetylene ratio $R$. Under the condition that other operating conditions remained unchanged, the ethylene selectivity, acetylene conversion rate and harmonic mean at different hydrogen/acetylene ratios were obtained, which were shown in Table 12.

Table 12. The optimization results of the second step. 


\begin{tabular}{cccc}
\hline $\boldsymbol{R}$ & $\boldsymbol{S}$ & $\boldsymbol{C}$ & $F 1$ \\
\hline 0.02 & 0.8947 & 0.1371 & 0.2378 \\
0.03 & 0.8599 & 0.2177 & 0.3474 \\
0.04 & 0.8207 & 0.3065 & 0.4463 \\
0.05 & 0.8051 & 0.4097 & 0.5431 \\
0.06 & 0.7646 & 0.5122 & 0.6135 \\
0.07 & 0.7294 & 0.6098 & 0.6643 \\
0.08 & 0.6985 & 0.7 & 0.6992 \\
0.09 & 0.6612 & 0.7642 & 0.709 \\
0.10 & 0.6308 & 0.813 & 0.7104 \\
0.11 & 0.5945 & 0.8525 & 0.7005 \\
0.12 & 0.5655 & 0.8852 & 0.6901 \\
0.13 & 0.534 & 0.9016 & 0.6707 \\
0.14 & 0.5065 & 0.9217 & 0.6537 \\
\hline
\end{tabular}

As can be seen from Table 12, when the hydrogen/acetylene ratio is $0.10, F 1$ reaches a maximum value of 0.7104 ; therefore, the optimal parameter combination is: temperature $318 \mathrm{~K}$, hydrogen/acetylene ratio 0.10 .

In the third step, we fixed the hydrogen/acetylene ratio $R$ at 0.10 to optimize $T$, and obtained the ethylene selectivity, acetylene conversion rate and harmonic mean at different temperatures with other operating conditions unchanged, which were shown in Table 13.

Table 13. The optimization results of the third step.

\begin{tabular}{cccc}
\hline T/K & $S$ & $C$ & $F 1$ \\
\hline 310 & 0.7473 & 0.6829 & 0.7137 \\
312 & 0.6917 & 0.7642 & 0.7261 \\
314 & 0.6562 & 0.8049 & 0.7230 \\
316 & 0.644 & 0.8211 & 0.7218 \\
318 & 0.6308 & 0.813 & 0.7104 \\
320 & 0.6293 & 0.813 & 0.7095 \\
322 & 0.6227 & 0.8049 & 0.7022 \\
324 & 0.6164 & 0.7967 & 0.6950 \\
326 & 0.6101 & 0.7886 & 0.6880 \\
328 & 0.6101 & 0.7886 & 0.6880 \\
\hline
\end{tabular}

As can be seen from Table 13, when the temperature is $312 \mathrm{~K}, F 1$ reaches a maximum value of 0.7261 ; therefore, the optimal parameter combination is: temperature $312 \mathrm{~K}$, hydrogen/acetylene ratio 0.10 .

In the fourth step, we fixed temperature $T$ at $312 \mathrm{~K}$ to optimize the hydrogen/acetylene ratio $R$. Under the condition that other operating conditions remained unchanged, the ethylene selectivity, acetylene conversion rate and harmonic mean at different hydrogen/acetylene ratios were obtained, which were shown in Table 14.

Table 14. The optimization results of the fourth step. 


\begin{tabular}{cccc}
\hline $\boldsymbol{R}$ & $\boldsymbol{S}$ & $\boldsymbol{C}$ & $F 1$ \\
\hline 0.02 & 0.916 & 0.0968 & 0.1751 \\
0.03 & 0.8571 & 0.1452 & 0.2483 \\
0.04 & 0.8581 & 0.2097 & 0.3370 \\
0.05 & 0.8537 & 0.2823 & 0.4243 \\
0.06 & 0.8178 & 0.3577 & 0.4977 \\
0.07 & 0.8081 & 0.4553 & 0.5824 \\
0.08 & 0.771 & 0.5528 & 0.6439 \\
0.09 & 0.7387 & 0.6667 & 0.7009 \\
0.10 & 0.6917 & 0.7642 & 0.7261 \\
0.11 & 0.6327 & 0.8443 & 0.7233 \\
0.12 & 0.5834 & 0.8934 & 0.7059 \\
0.13 & 0.5465 & 0.9189 & 0.6854 \\
0.14 & 0.5146 & 0.9361 & 0.6641 \\
\hline
\end{tabular}

As can be seen from Table 14, when the hydrogen/acetylene ratio is $0.10, F 1$ reaches a maximum value of 0.7261 ; therefore, the optimal parameter combination is: temperature $312 \mathrm{~K}$, hydrogen/acetylene ratio 0.10 .

We can conclude that the optimization results of the fourth step are the same as that of the third step; hence, the whole iterative process is terminated, and the optimal parameter combination is: temperature $312 \mathrm{~K}$ and hydrogen/acetylene ratio 0.10 .

\subsubsection{Analysis of Optimized Results}

Taking the comprehensive performance metrics of ethylene selectivity and acetylene conversion rate as the optimization objective, we can obtain the optimal temperature of $312 \mathrm{~K}$ and the hydrogen/acetylene ratio of 0.10 (molar ratio 1.3). Under the condition of optimal temperature and hydrogen/acetylene ratio, the selectivity is $69.17 \%, 1.26 \%$ lower than that before optimization, the conversion rate is $76.42 \%, 10.57 \%$ higher than that before optimization, and $F 1$ is $72.61 \%, 4.55 \%$ higher than that before optimization. From the comprehensive comparison, although the selectivity is reduced marginally, the conversion rate and F1 are improved greatly, and the comprehensive performance of hydrogenation reaction is better than that before optimization.

Table 15 shows the comparison between the optimized simulation inlet and outlet data and the actual inlet and outlet data. It can be seen from Table 15 that the optimized simulation outlet temperature, pressure and flow rate are basically consistent with the actual outlet data. The molar fractions of ethane, acetylene and hydrogen decrease by $0.13 \%, 0.23 \%$ and $0.15 \%$ respectively compared with the actual outlet data, while the mole fraction of ethylene increases by $0.51 \%$ compared with the actual outlet data. From the comprehensive comparison, the optimized comprehensive performance is better than that before optimization. With other conditions unchanged, by optimizing the inlet temperature and hydrogen/acetylene ratio, we can improve the comprehensive performance of C-2 hydrogenation adiabatic reaction, which shows great economic application value and can provide some guidance for industrial practice.

Table 15. Comparison of optimized simulation inlet and outlet data and actual inlet and outlet data. 


\begin{tabular}{ccccc}
\hline Items & $\begin{array}{c}\text { Actual } \\
\text { Inlet }\end{array}$ & $\begin{array}{c}\text { Inlet After } \\
\text { Optimization }\end{array}$ & $\begin{array}{c}\text { Actual } \\
\text { Outlet }\end{array}$ & $\begin{array}{c}\text { Outlet After } \\
\text { Optimization }\end{array}$ \\
\hline Temperature/K & 320 & 312 & 351 & 352 \\
Pressure/MPa & 1.75 & 1.75 & 1.75 & 1.75 \\
Mass flow/kg/s & 51.8721 & 51.8721 & 51.8721 & 51.8204 \\
$\mathrm{C}_{2} \mathrm{H}_{6} / \mathrm{mol} \%$ & 20.63 & 20.55 & 21.22 & 21.09 \\
$\mathrm{C}_{2} \mathrm{H}_{4} / \mathrm{mol} \%$ & 76.96 & 76.63 & 77.88 & 78.39 \\
$\mathrm{C}_{2} \mathrm{H}_{2} / \mathrm{mol} \%$ & 1.23 & 1.23 & 0.52 & 0.29 \\
$\mathrm{H}_{2} / \mathrm{mol} \%$ & 1.18 & 1.59 & 0.38 & 0.23 \\
\hline
\end{tabular}

\subsubsection{Optimization of Hydrogen Load Distribution}

The previous optimization is aimed at the optimization of the first section reactor. C-2 hydrogenation reactor is composed of three section reactor. Now the total amount of hydrogen was kept constant to optimize the hydrogen load distribution ratio of each section. According to the actual industrial data, we can know that the amount of hydrogen in the first section was greater than that in the second section, and the amount of hydrogen in the second section was greater than that in the third section, that is, the amount of hydrogen in each section was gradually decreasing. Under this assumption, there were eight eligible distribution schemes, namely 4:3:3, 4:4:2, 5:3:2, 5:4:1, 6:2:2, 6:3:1, $7: 2: 1,8: 1: 1$.

On the premise that the outlet acetylene concentration meets the requirement, various performance metrics under different hydrogen load distribution ratios were obtained, and the optimization results were shown in Table 16.

Table 16. The optimizing results under different hydrogen load distribution ratios.

\begin{tabular}{cccc}
\hline H1:H2:H3 & $S$ & $C$ & $F 1$ \\
\hline $4: 3: 3$ & 0.7044 & 0.99998 & 0.8266 \\
$4: 4: 2$ & 0.7186 & 0.9999 & 0.8362 \\
$5: 3: 2$ & 0.7166 & 0.9999 & 0.8349 \\
$5: 4: 1$ & 0.7023 & 0.9996 & 0.8250 \\
$6: 2: 2$ & 0.6955 & 0.9995 & 0.8202 \\
$6: 3: 1$ & 0.6972 & 0.9988 & 0.8212 \\
$7: 2: 1$ & 0.6792 & 0.994 & 0.8070 \\
$8: 1: 1$ & 0.6481 & 0.9669 & 0.7760 \\
\hline
\end{tabular}

As can be seen from Table 16, on the premise that the outlet acetylene concentration meets the requirement, that is, when the conversion rate is greater than $99 \%$, the maximum ethylene selectivity can be obtained when $\mathrm{H} 1: \mathrm{H} 2: \mathrm{H} 3$ is 4:4:2, and meanwhile, the comprehensive performance metrics $F 1$ of ethylene selectivity and acetylene conversion rate is also optimal.

The performance metrics after optimization and those before optimization are shown in Table 17.

Table 17. Comparison of various performance metrics after optimization and those before optimization.

\begin{tabular}{ccccc}
\hline Performance Metrics & $S$ & $C$ & $F 1$ & $Y$ \\
\hline Before optimization & 0.6669 & 1 & 0.8002 & 0.0107 \\
After optimization & 0.7186 & 0.9999 & 0.8362 & 0.0189 \\
\hline
\end{tabular}

In Table 17, $S$ stands for ethylene selectivity, $C$ stands for acetylene conversion rate, $F 1$ stands for comprehensive performance metrics of ethylene selectivity and acetylene conversion rate, and $Y$ stands for ethylene yield. As can be seen from Table 17, under the condition that the outlet acetylene concentration meets the requirement, the optimized ethylene selectivity is $71.86 \%, 5.17 \%$ higher than that before optimization, the comprehensive performance metrics are $83.62 \%, 3.60 \%$ 
higher than that before optimization, and the ethylene yield is $1.89 \%, 0.82 \%$ higher than that before optimization. In general, the performance metrics after optimization are improved to some extent compared with that before optimization.

Under the condition that the structure of the reactor and other inlet conditions remain unchanged, by optimizing the hydrogen load distribution ratio of each section of the reactor, the performance metrics of the adiabatic reaction of $\mathrm{C}-2$ hydrogenation are improved, which shows great economic application value and can provide some guidance for industrial practice.

\subsection{Ethylene Selectivity Analysis}

According to the optimization results of the first step and the third step in Section 3.4.2, the changing curve of ethylene selectivity with inlet temperature at different hydrogen/acetylene ratios was obtained, which was shown in Figure 9. It can be seen from the figure that, under the condition of a certain hydrogen/acetylene ratio, ethylene selectivity decreases with the increase of inlet temperature, which is consistent with the general law of reaction performance of catalyst, namely, with the increase of temperature, the activity increases while the selectivity decreases.

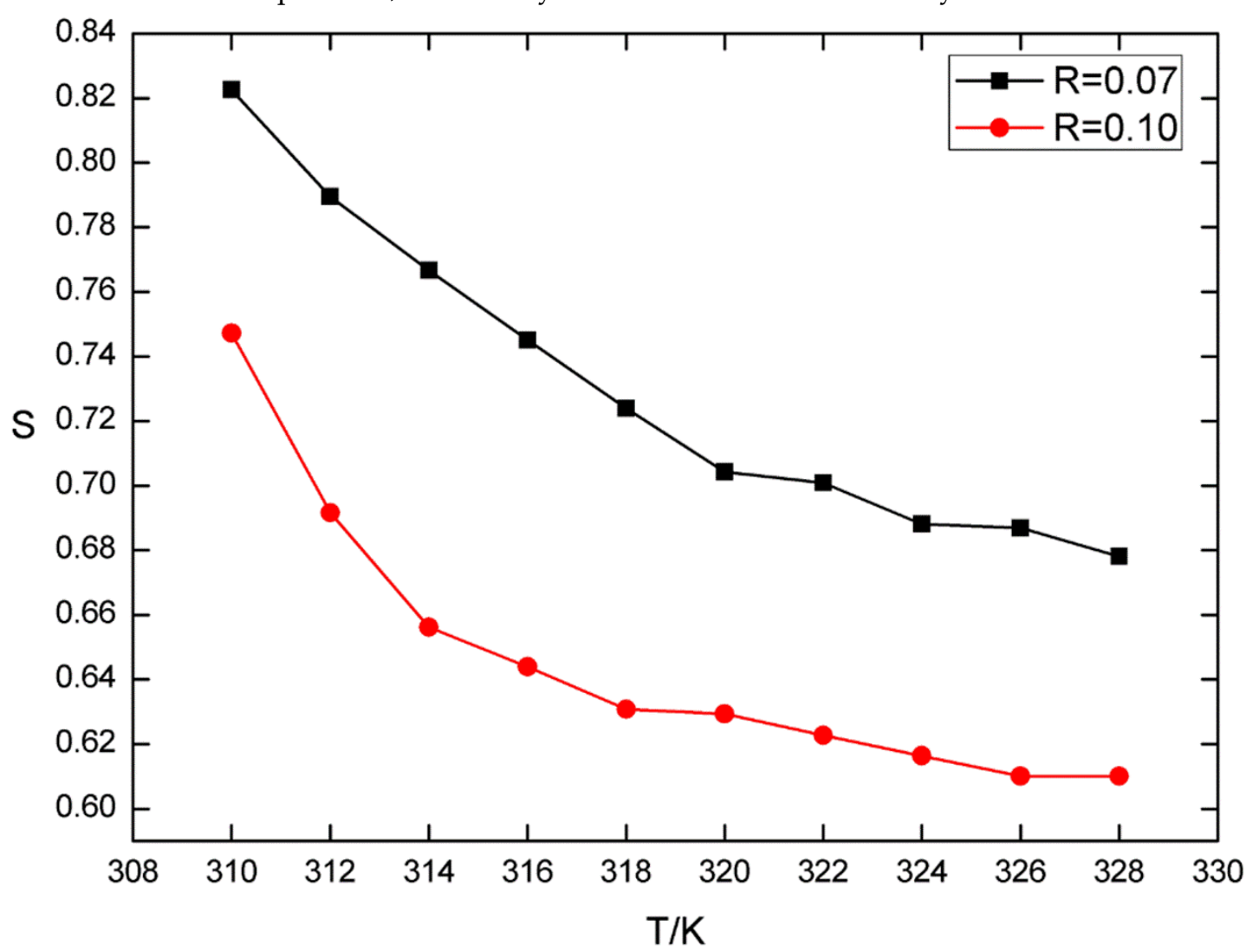

Figure 9. The effect of temperature on the ethylene selectivity.

According to the optimization results of the second step and the fourth step in Section 3.4.2, the changing curve of ethylene selectivity with hydrogen/acetylene ratio at different temperatures was obtained, which was shown in Figure 10. It can be seen from the figure that, at a certain temperature, ethylene selectivity decreases with the increase of hydrogen/acetylene ratio. Theoretical analysis is as follows: the number of moles of acetylene in the reaction is fixed, when the hydrogen/acetylene ratio increases, that is, when the hydrogen concentration increases, side reaction intensifies and ethylene hydrogenates to ethane; hence, the selectivity decreases. Of course, in order to make acetylene hydrogenation index, namely, the outlet acetylene concentration less than $5 \mathrm{ppm}$, the hydrogen concentration in the reaction should be controlled in the appropriate range, and the control of reaction temperature should also be adjusted according to the reaction performance of the catalyst and the specific situation during use, whose purpose is to produce qualified hydrogenation products, obtain the highest ethylene yield, and have a long service cycle of catalyst. 


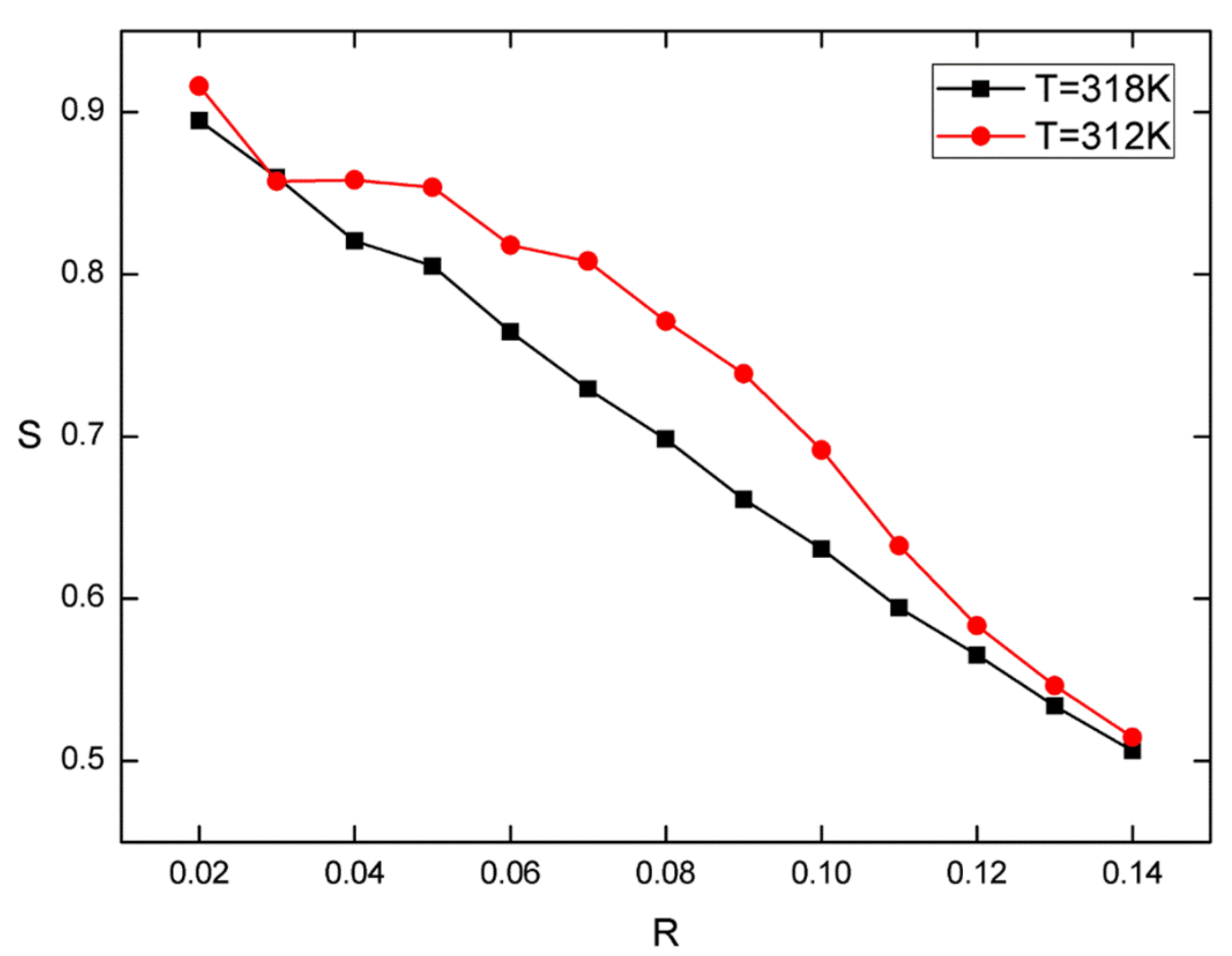

Figure 10. The effect of hydrogen/acetylene ratio on the ethylene selectivity.

In the book Ethylene Process and Technology [19], Wang and He (2000) gives an empirical mathematical model of selectivity, that is:

$$
S=0.035 \mathrm{e}^{(2397.07-2.58 D) / T} c_{A 0}^{0.12} c_{H 0}^{-0.09} V^{2.41}
$$

where, $s$ stands for selectivity, $D$ stands for catalyst service time, $T$ stands for reaction temperature, $c_{A 0}$ stands for acetylene concentration, $c_{H_{0}}$ stands for hydrogen concentration, and $V$ stands for space velocity.

It can be seen from Equation (18) that, under the condition that the catalyst service time, acetylene concentration and space velocity are fixed, the selectivity decreases with the increase of reaction temperature, as well as hydrogen concentration, which is consistent with the above analysis.

\subsection{Acetylene Conversion Rate Analysis}

According to the optimization results of the first step and the third step in Section 3.4.2, the changing curve of acetylene conversion rate with inlet temperature at different hydrogen/acetylene ratios was obtained, which was shown in Figure 11. As can be seen from the figure, when the inlet temperature is not too high, the conversion rate increases with the increase of temperature; when the temperature exceeds a certain value, the conversion rate decreases with the increase of temperature. Theoretical analysis is as follows: when the temperature is not too high, acetylene hydrogenation reaction plays a leading role; with the increase of temperature, the reaction of acetylene hydrogenation to ethylene is violent; hence, the amount of ethylene rapidly increases, the corresponding amount of acetylene quickly decreases, namely acetylene conversion rate increases. When the temperature exceeds a certain value, ethylene hydrogenation reaction plays a leading role; with the increase of temperature, the reaction of ethylene hydrogenation to ethane is violent, while acetylene hydrogenation reaction is very weak; hence, the amount of ethylene generated is very small, and the corresponding amount of acetylene converted is very small; thus, the acetylene conversion rate decreases. 


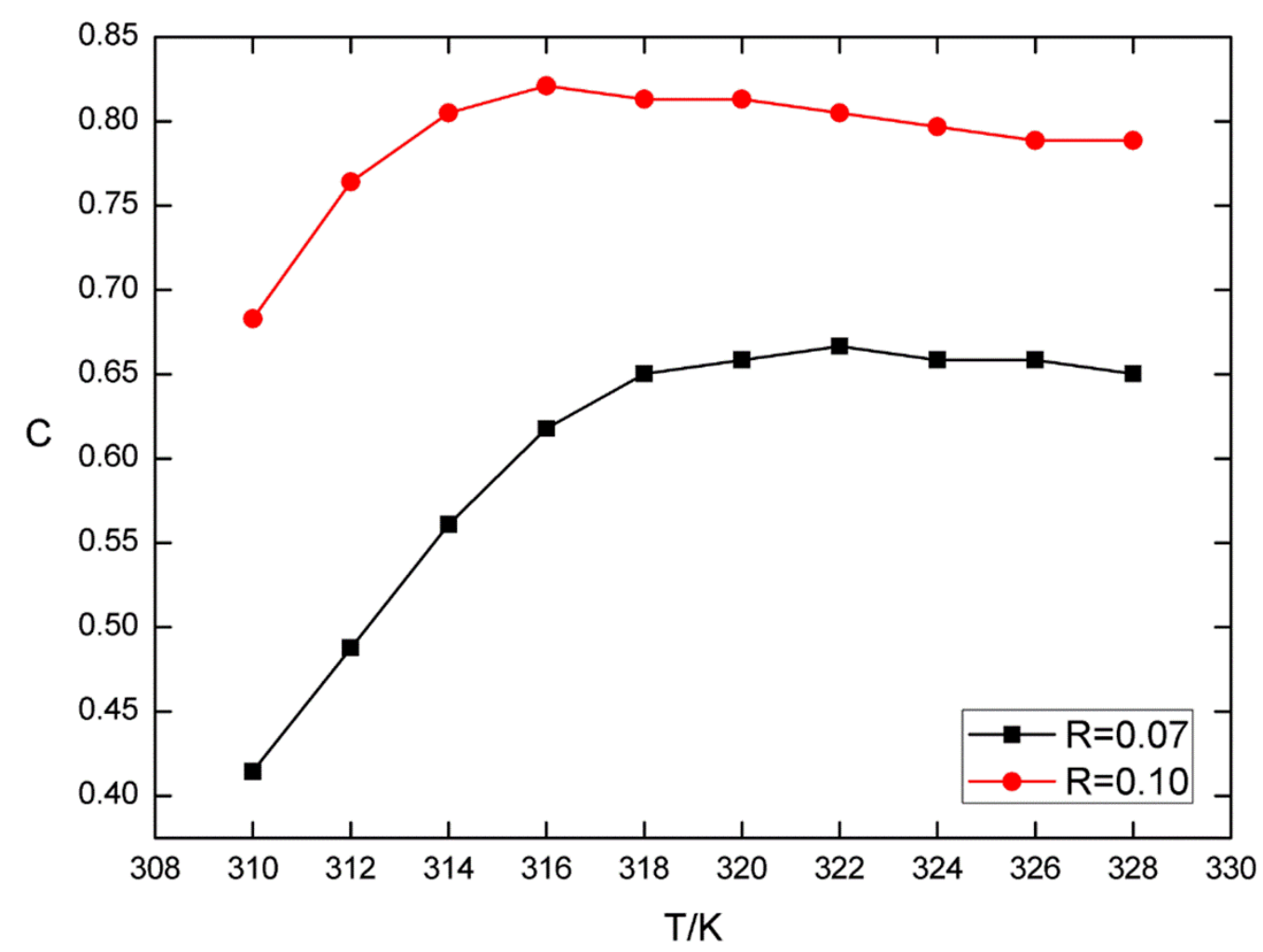

Figure 11. The effect of temperature on the acetylene conversion rate.

According to the optimization results of the second step and the fourth step in Section 3.4.2, the changing curve of acetylene conversion rate with hydrogen/acetylene ratio at different temperatures was obtained, which was shown in Figure 12. As can be seen from the figure, when the temperature is fixed, the acetylene conversion rate increases gradually with the increase of the hydrogen/acetylene ratio. Theoretical analysis is as follows: the higher the hydrogen/acetylene ratio is, the higher the hydrogen concentration is, the more violent the acetylene hydrogenation reaction is, the greater the amount of ethylene generated is, and the greater the amount of acetylene converted is; hence, the acetylene conversion rate increases. 


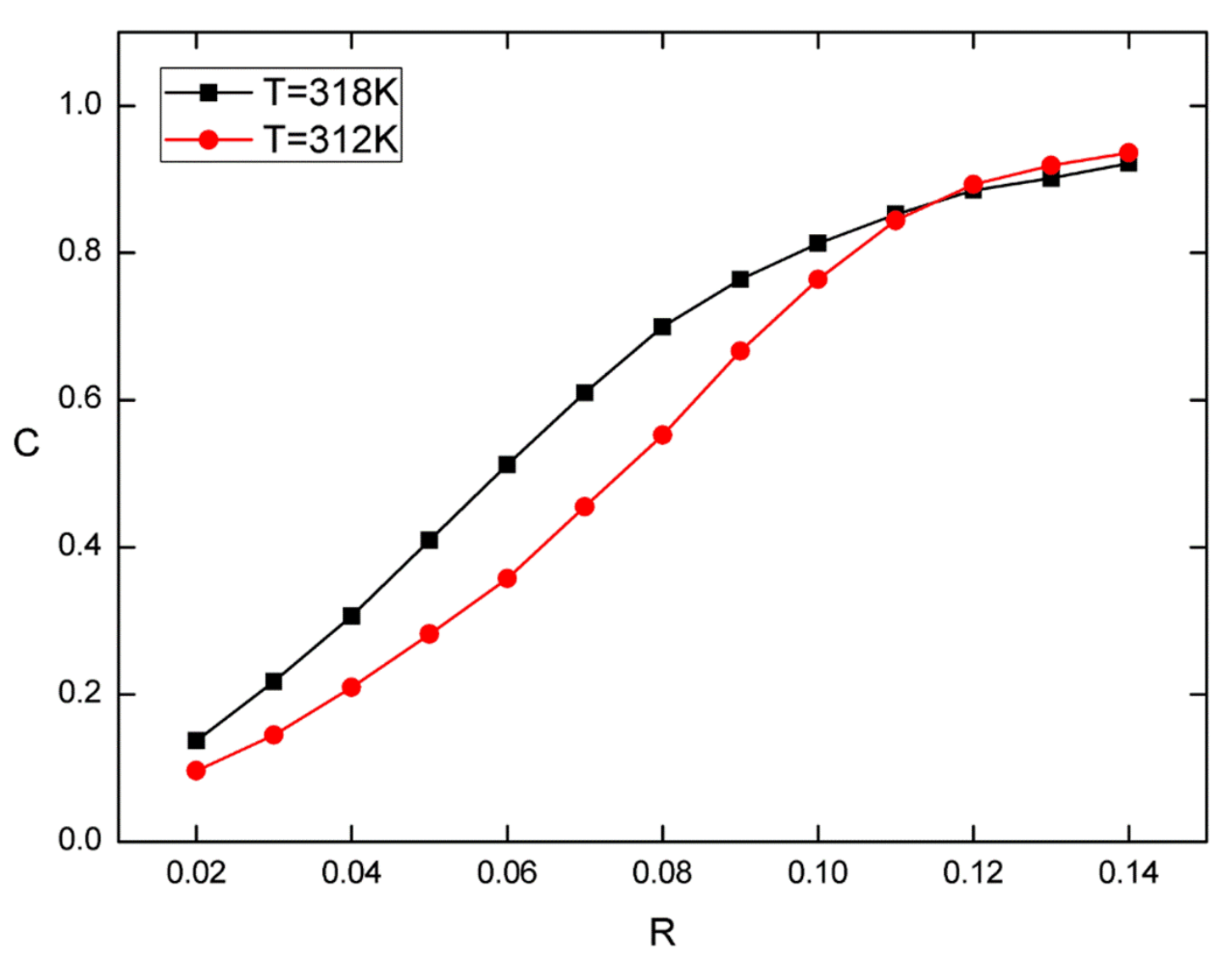

Figure 12. The effect of hydrogen/acetylene ratio on the acetylene conversion rate.

According to the previous analysis, the changing trend of ethylene selectivity with temperature and hydrogen/acetylene ratio is different from that of acetylene conversion rate with temperature and hydrogen/acetylene ratio. The selectivity decreases with the increase of temperature and hydrogen/acetylene ratio. The conversion rate first increases and then decreases with the increase of temperature, and increases with the increase of hydrogen/acetylene ratio. In order to maximize the selectivity, temperature and hydrogen/acetylene ratio should take the minimum values. However, the conversion rate at this time is very small and cannot meet the requirement of outlet acetylene concentration. On the contrary, in order to maximize the conversion rate, larger values should be taken for temperature and hydrogen/acetylene ratio. However, the selectivity at this time is very small, ethylene loss is serious, and economic benefits become worse. In other words, selectivity and conversion rate do not both get the optimal value at the same temperature and hydrogen/acetylene ratio. In order to obtain the optimal temperature and hydrogen/acetylene ratio, a third evaluation index $F 1$ was defined, which was the harmonic mean of selectivity and conversion rate. Then, the single factor test rotation method was adopted to maximize the optimization goal $F 1$, and the optimal temperature and hydrogen/acetylene ratio were obtained: temperature $312 \mathrm{~K}$, hydrogen/acetylene ratio 0.10 (molar ratio 1.3). Through the comparison and analysis with the inlet and outlet data before optimization, it is found that the optimized temperature and hydrogen/acetylene ratio greatly improve the comprehensive performance of C-2 hydrogenation reaction, which shows some economic application value.

\section{Conclusions}

In this paper, the simulation and optimization of the C-2 hydrogenation adiabatic reactor of an actual ethylene production unit in a chemical plant were performed by CFD. The mathematical model of the C-2 hydrogenation adiabatic reaction process was established, and then the reaction process was accurately simulated by CFD, obtaining the distribution law of each physical field in the reactor, and finally the parameters in the reaction process, such as the structure of the reactor, the inlet 
temperature, the hydrogen/acetylene ratio, etc., were optimized, which improves the comprehensive performance of ethylene selectivity and acetylene conversion rate.

The main work of this paper is summarized as follows:

(1) Aiming at the mechanism of C-2 hydrogenation reaction, a kinetic model based on the Arrhenius formula was established. The $\mathrm{C}-2$ hydrogenation reaction was a catalytic hydrogenation reaction and there were both gaseous phase components and solid phase catalysts in the reaction region; hence, the porous medium model was established to simulate the flow and heat transfer of the fluid in the reaction region. Based on the traditional equations of fluid mechanics, the governing equations of the porous medium model were established.

(2) Based on the established mathematical model, the CFD simulation software ANSYS Fluent 14.5 was employed for simulating the reaction process accurately. The finite rate chemical reaction model was used for the chemical reaction model and Laminar Finite-Rate model was used for the turbulence-chemistry interaction model. The inlet boundary conditions were determined as the mass flow inlet boundary, the outlet boundary conditions were determined as the pressure outlet boundary, and the wall condition was determined as wall type, the shear condition was determined as the no-slip boundary condition. The CFD algorithm adopted SIMPLE algorithm. In the results and discussion, the convergence of the model was analyzed, the accuracy of the model was validated, and the distribution law of each physical field in the reactor was analyzed.

(3) On the basis of CFD simulation, the structure and operating conditions of the reactor were optimized with the optimization objective of the comprehensive performance of ethylene selectivity and acetylene conversion rate. Under the same inlet conditions, the change of length/diameter ratio has a marginal effect on the reaction selectivity, conversion rate and harmonic mean of the adiabatic reactor. The operating conditions were optimized by the single factor test rotation method, obtaining an optimal set of temperature and hydrogen/acetylene ratio. The selectivity, conversion rate, and comprehensive performance after and before optimization were analyzed. Hydrogen load distribution was optimized under the condition that the total amount of hydrogen was fixed. Finally, the changing trends of ethylene selectivity and acetylene conversion rate with inlet temperature and hydrogen/acetylene ratio were given. By optimizing the structure and operating conditions of the reactor, as well as hydrogen load distribution, the comprehensive performance metrics of the C-2 hydrogenation reaction have been significantly improved compared with that before optimization, which can provide some guidance for the actual industrial production process.

Funding: This research received no external funding.

Acknowledgments: We thank the reviewers for helpful suggestions and comments on the manuscript or study.

Conflicts of Interest: The authors declare no conflict of interest.

\section{References}

1. Tian, L.; Hu, G.; Du, W.; Qian, F. Comprehensive CFD simulation of the optimizations of geometric structures and operating parameters for industrial acetylene hydrogenation reactors. Can. J. Chem. Eng. 2016, 94, 2427-2435, doi:10.1002/cjce.22627.

2. Azizi, M.; Zolfaghari Sharak, A.; Mousavi, S.A.; Bakhtiari Ziabari, F.; Shariati, J.; Azizi, S. Study on the Acetylene Hydrogenation Process for Ethylene Production: Simulation, Modification, and Optimization. Chem. Eng. Commun. 2013, 200, 863-877, doi:10.1080/00986445.2012.721033.

3. $\mathrm{Xu}, \mathrm{X}$;; Zhao, $\mathrm{Z}$. Modelling and minimum variance covariance constrained control of acetylene hydrogenation industrial device. In Proceedings of The 27th Chinese Control and Decision Conference (2015 CCDC), Qingdao, China, 23-25 May 2015; pp. 3196-3203.

4. Lu, Q.; Yang, Y.; Tan, D.; Li, B.; Liu, H.; Lu, L.; Liu, M. Simulation and optimization of process conditions of C2 hydrogenation side line in ethylene unit. Petrochemical Technology \& Application 2016, 34, 395-398, doi:10.3969/j.issn.1009-0045.2016.05.010.

5. Zhang, D.; Wang, G. Simulating and Analysis of Reactor for Selective Hydrogenation of Acetylene. PETROCHEMICAL TECHNOLOGY 2003, 32, 414-417, doi:10.3321/j.issn:1000-8144.2003.05.013. 
6. Yang, F.; Zhang, L.; Xie, C. Analysis on Optimized Operation of Acetylene Converter. Contemporary Chemical Industry 2011, 40, 1007-1012, doi:10.3969/j.issn.1671-0460.2011.10.005.

7. Zhong, Z. Real-time Evaluation Method for the selectivity of Acetylene Hydrogenation Catalyst and Its Application. ETHYLENE INDUSTRY 2005, 17, 60-62, doi:10.3969/j.issn.1671-7120.2005.04.016.

8. Peng, H. Effect of operating parameters of $\mathrm{C} 2$ hydrogenation catalyst on hydrogenation reaction and its optimization. Ethylene Industry 2014, 26, 60-64, doi:10.3969/j.issn.1671-7120.2014.02.023.

9. Che, C.; Cheng, L.; Gu, L.; Han, W.; Xie, W.; Qian, Y.; Tan, D.; Chang, X.; Zhang, F. Effects of Process Conditions on Performance of C2 Front-end Hydrogenation Catalyst. Contemporary Chemical Industry 2013, 42, 1207-1209, doi:10.3969/j.issn.1671-0460.2013.09.002.

10. Dong, X. Analyzed the effects of $\mathrm{C} 2$ hydrogenation reactor in Daqing Petrochemical Company. Jiangxi Chemical Industry 2014, 0, 85-88, doi:10.3969/j.issn.1008-3103.2014.02.026.

11. Huang, W. Commercial application and operation optimization of C2 hydrogenation catalyst KL7741B-T. INDUSTRIAL CATALYSIS 2016, 24, 58-63, doi:10.3969/j.issn.1008-1143.2016.08.011.

12. Gu, H.; Zhang, L.; Zhang, Y.; Zhou, L.; Zhou, J. Influence of CO fluctuation on the C2 front-end hydrogenation reaction and its countermeasures. Ethylene Industry 2017, 29, 24-28, doi:10.3969/j.issn.16717120.2017.04.007.

13. Jiang, M.; Wang, J.; Han, F. Optimization of operation performance of C2 hydrogenation reactor. QILU PETROCHEMICAL TECHNOLOGY 2002, 30, 305-307.

14. Hu, B.; Hu, G.; Jiang, D.; Qian, F. Simulation and optimization of operating parameters of isothermal reaction process for acetylene hydrogenation. CIESC Journal 2015, 66, 366-372.

15. Tian, L.; Jiang, D.; Qian, F. Simulation and optimization of acetylene converter with decreasing catalyst activity. CIESC Journal 2012, 63, 185-192, doi:10.3969/j.issn.0438-1157.2012.01.026.

16. Wang, F.; Luo, N.; Jiang, D.; Qian, F. Dynamic simulation and control of acetylene hydrogenation reactor in ethylene plant. Computers and Applied Chemistry 2012, 29, 85-89, doi:10.3969/j.issn.1001-4160.2012.01.018.

17. Du, W.; Bao, C.; Chen, X. Dynamic Optimization of Tandem Acetylene Hydrogenation Process. Information and Control 2017, 46, 83-89, doi:10.13976/j.cnki.xk.2017.0083.

18. Hu, B. CFD Simulation and Operation Parameters Optimization of C2 Hydrogenation Isothermal Reaction Process. M.E. Thesis, East China University of Science and Technology, Shanghai, China, 2016.

19. Wang, S.; He, X. Ethylene process and technology, 1st ed.; China Petrochemical Press: Beijing, China, 2000; pp. 501-545. 